\title{
Controlling Heterogeneity and Increasing Titer from Riboswitch-Regulated Bacillus subtilis Spores for Time-Delayed Protein Expression Applications
}

By Denis Tamiev ${ }^{1}$, Alyssa Lantz ${ }^{1}$, Grace Vezeau², Howard Salis ${ }^{2}$, and Nigel F. Reuel ${ }^{3 *}$

1. Department of Biochemistry Biophysics and Molecular Biology, lowa State University

2. Department of Chemical Engineering, Agricultural and Biological Engineering, Penn State University.

3. Department of Chemical and Biological Engineering, lowa State University

* Corresponding Author-reuel@iastate.edu

\section{Contents}

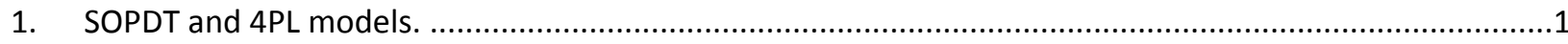

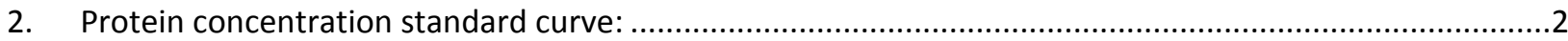

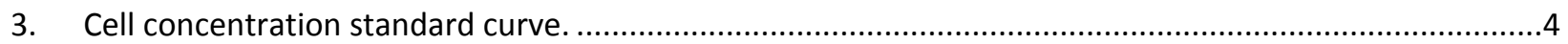

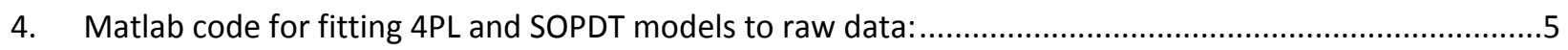

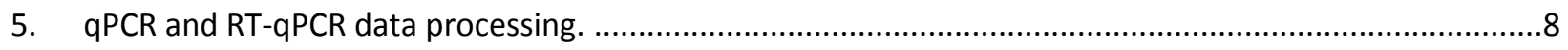

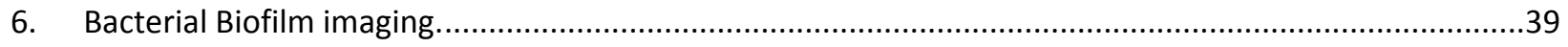

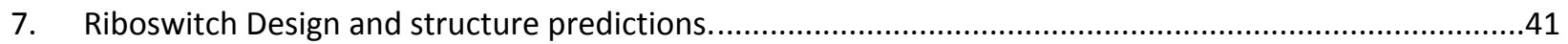

\section{SOPDT and 4PL models.}

Cellular growth as a measure of OD at $600 \mathrm{~nm}$ was recorded and fitted with a 4 parameter logistics (4PL) function. Expression of RFP was measured as a function of fluorescence of RFP (584/607nm excitation/emission wavelength respectively) and fitted with a second order plus dead time function (SOPDT). Fit parameters (constant values of each function) were recorded for the following experiments:

(1) B. subtilis strains 168 , PolYB- or PolYA- that carried either a theta replicating $(\mathrm{pHT})$ or integrating (pBS1C) DNA plasmid (Fig. 4).

(2) B. subtilis strains 168 , PolYB- or PolYA- that carried a theta replicating plasmid ( $\mathrm{pHT}$ ) were subjected to UV treatment of various lengths (Fig. 9).

(3) B. subtilis strains 168, PolYB- or PolYA- that carried a theta replicating plasmid (pHT) were subjected to sporulation (Fig. 7). 


\section{Protein concentration standard curve:}

In order to convert RFU data to meaningful units, we created a standard curve using Pierce660 assay (Thermo Fisher Scientific). In this assay, a BSA standard protein solution (sigma) was diluted according to Supplement Table 1. The protein sample was mixed with the Pierce660 assay master mix according to the manufacturer's protocol. Absorbance values at $660 \mathrm{~nm}$ were measured and correlated with protein concentration (Supplement Figure 1). In order to correlate protein concentration with fluorescence, RFP was purified as described in the methods section. Fluorescence of the purified protein as well as absorbance at $660 \mathrm{~nm}$ were measured (<modelParam.xlsx > and Supplement Figure 2, Supplement Table 2). As a result, a standard curve that correlated RFP fluorescence (RFU) to protein concentration ( $\mathrm{mg} / \mathrm{ml}$ ) through absorbance at $660 \mathrm{~nm}$ (RAU) was created. All measurements were performed on a 96-well plate with a BioTek Synergy Neo 2 plate reader.

Supplement Table 1 - Dilution table of BSA standard protein solution used in Pierce660 assay (supplement figure 1).

\begin{tabular}{|c|c|c|c|c|c|}
\hline Sample & $\begin{array}{c}\text { Concentration } \\
(\mathrm{mg} / \mathrm{ml})\end{array}$ & AVG A660nm & \multicolumn{3}{|c|}{ A660nm } \\
\hline 1 & 1 & 0.456 & 0.445 & 0.462 & 0.462 \\
\hline 2 & 0.5 & 0.336 & 0.34 & 0.331 & 0.339 \\
\hline 3 & 0.25 & 0.272 & 0.269 & 0.274 & 0.273 \\
\hline 4 & 0.125 & 0.237 & 0.232 & 0.238 & 0.242 \\
\hline 5 & 0.0625 & 0.217 & 0.222 & 0.213 & 0.216 \\
\hline 6 & 0.03125 & 0.203 & 0.204 & 0.197 & 0.208 \\
\hline 7 & 0.015625 & 0.197 & 0.201 & 0.197 & 0.193 \\
\hline 8 & 0.0078125 & 0.195 & 0.198 & 0.194 & 0.193 \\
\hline 9 & 0.00390625 & 0.191 & 0.197 & 0.189 & 0.188 \\
\hline 10 & 0.001953125 & 0.193 & 0.197 & 0.194 & 0.189 \\
\hline & Inknown & 0.256 & 0.257 & 0.256 & 0.255 \\
\hline
\end{tabular}


Supplement Table 1 - Fluorescence (RFU) and corresponding protein concentration $(\mathrm{mg} / \mathrm{ml}$ ) values of purified RFP sample used in Pierce660 assay (Supplement figure 2).

\begin{tabular}{|c|c|c|c|}
\hline Concentration $(\mathrm{mg} / \mathrm{ml})$ & AVG RFU & \multicolumn{2}{|c|}{ RFU } \\
\hline 0.0518 & 12041.5 & 12481 & 11602 \\
\hline 0.0259 & 5648.5 & 5744 & 5553 \\
\hline 0.0129 & 2684 & 2750 & 2618 \\
\hline 0.00649 & 1327 & 1331 & 1323 \\
\hline 0.00324 & 593.5 & 615 & 572 \\
\hline 0.00162 & 279.5 & 279 & 280 \\
\hline 0.00162 & 269.5 & 273 & 266 \\
\hline 0 & 28 & 30 & 26 \\
\hline
\end{tabular}

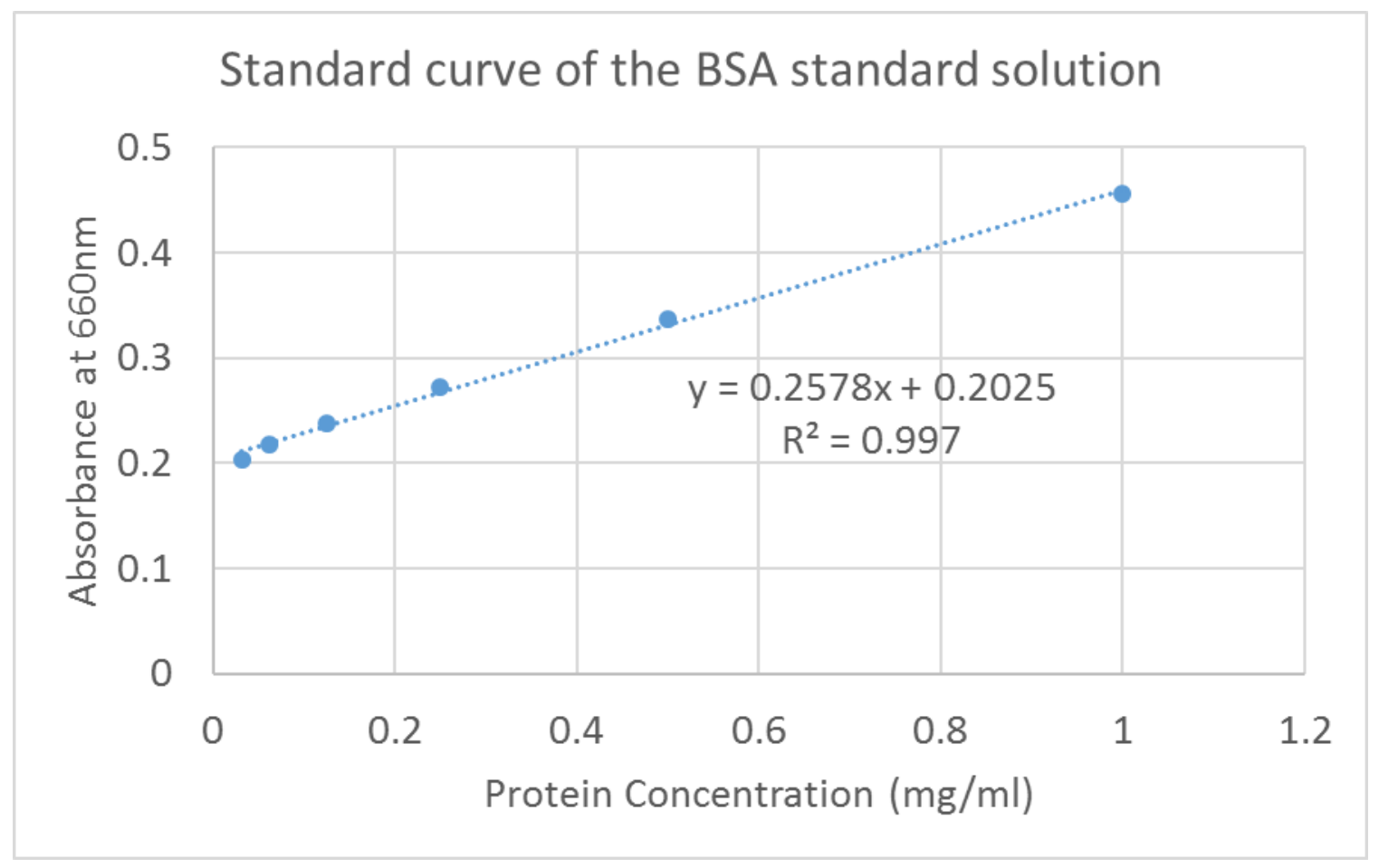

Supplement figure 1 - Protein concentration of the BSA standard protein solution and absorbance at 660nm standard curve (Pierce660 assay). 


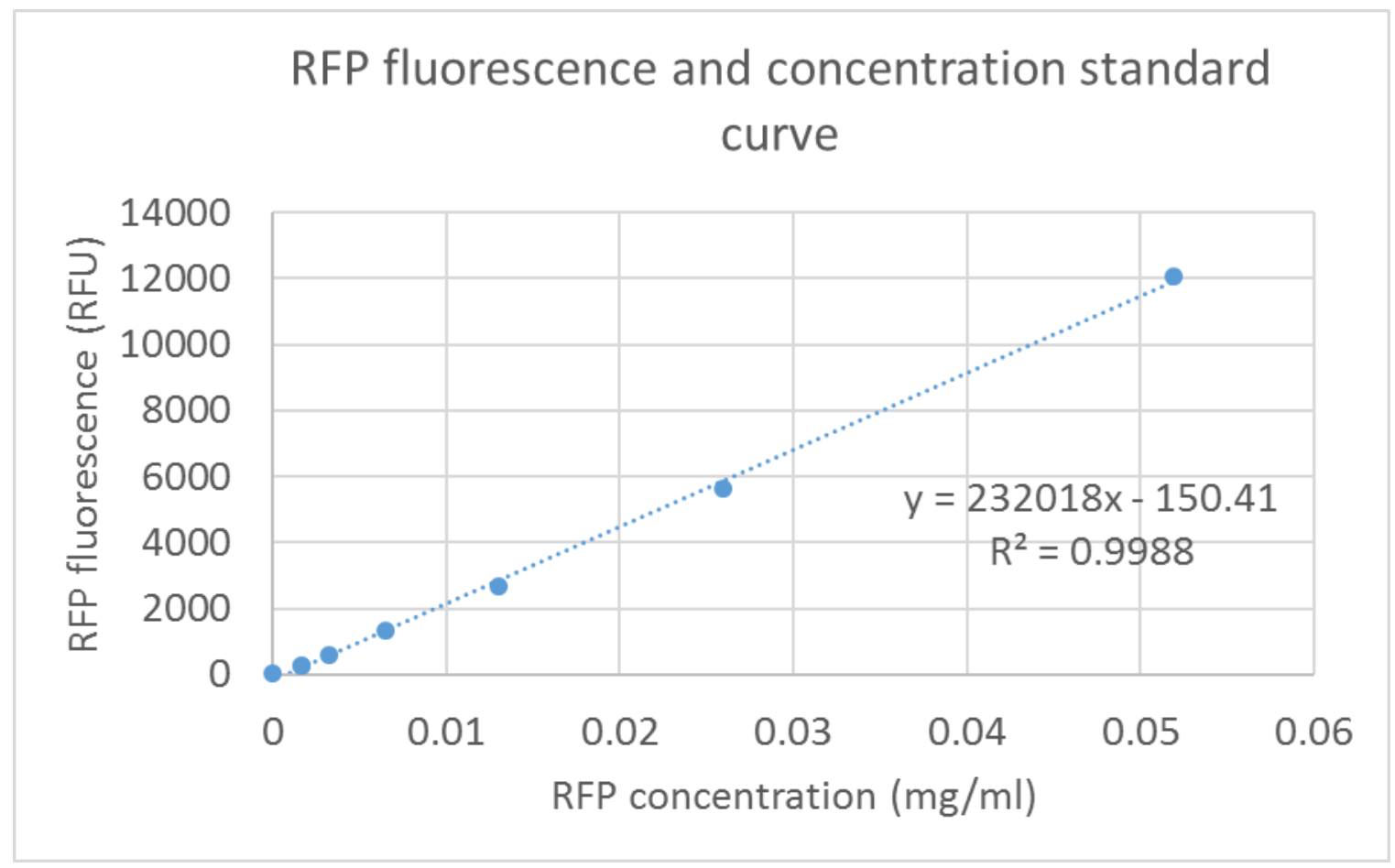

Supplement figure 2 Fluorescence and protein concentration of RFP standard curve.

\section{Cell concentration standard curve.}

B. subtilis 168 cells were cultured in the plate reader, and the concentrations of cells in cultures were approximated using absorbance at $600 \mathrm{~nm}$. In order to convert the absorbance values to meaningful concentration, cell count, flow cytometry was used. Cell samples were prepared as described in the method section.

First, absorbance at $600 \mathrm{~nm}$ acquired from with the plate reader was converted experimentally to absorbance values with a constant $1 \mathrm{~cm}$ path length, at the same, $600 \mathrm{~nm}$, wavelength (Supplement figure 3 ). Then, $B$. subtilis cell culture was diluted to concentrations that corresponded with OD600 values of 0.004 through 0.014 , as these concentrations were optimal for performing flow cytometry assays. A standard curve of cell count vs optical density at $600 \mathrm{~nm}$ with path length of $1 \mathrm{~cm}$ was created (supplement figure 4). 


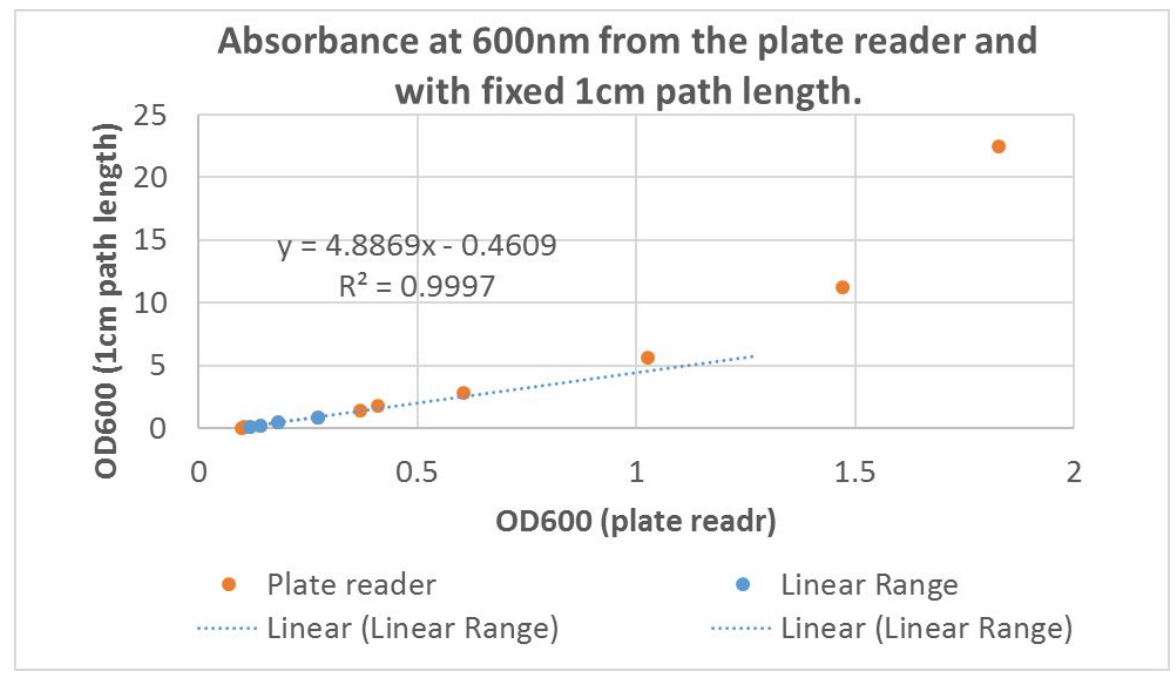

Supplement figure 3 - Standard curve of absorbance at 600nm acquired with a plate reader (96-well plate) and a $1 \mathrm{~cm}$ path length cuvette.

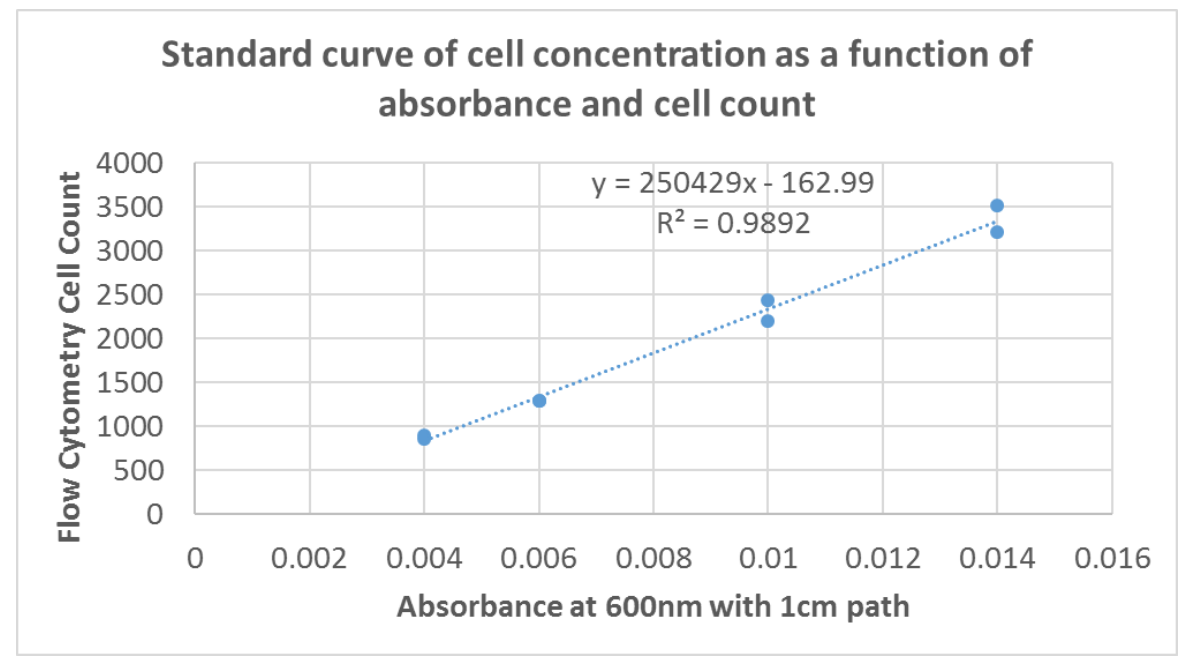

Supplement figure 4 - Standard curve of cell concentration as a function of absorbance at 600nm with a $1 \mathrm{~cm}$ path length, and cell count (flow cytometry).

\section{Matlab code for fitting 4PL and SOPDT models to raw data:}

Below is a sample code that was used to (1) reduce absorbance and fluorescence data to 4PL and SOPDT models, (2) extract LKPmax and LAG model parameters, and (3) generate normal distribution plots from LKPmax and LAG.

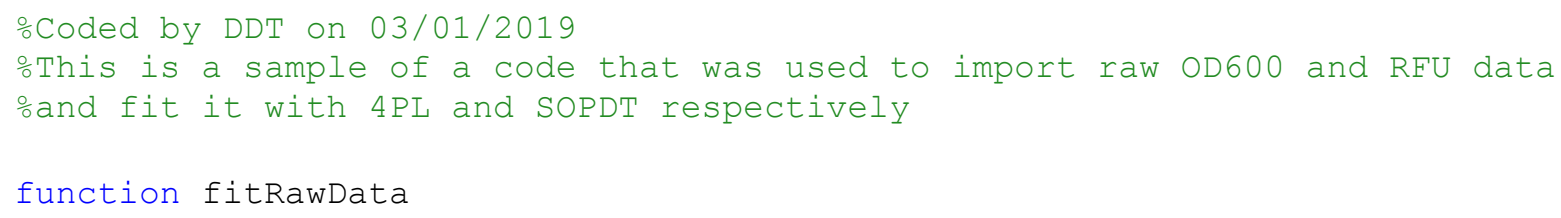




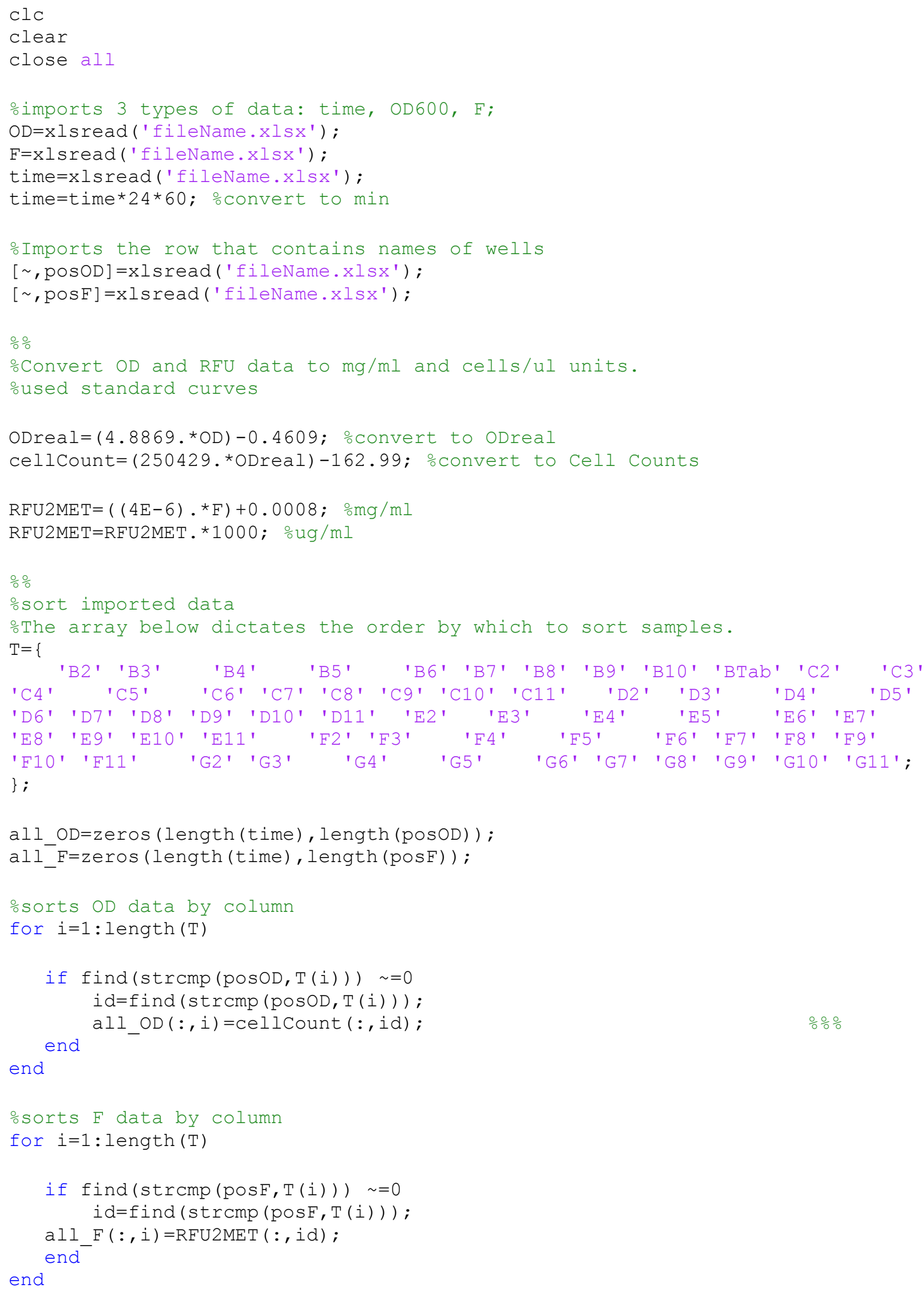




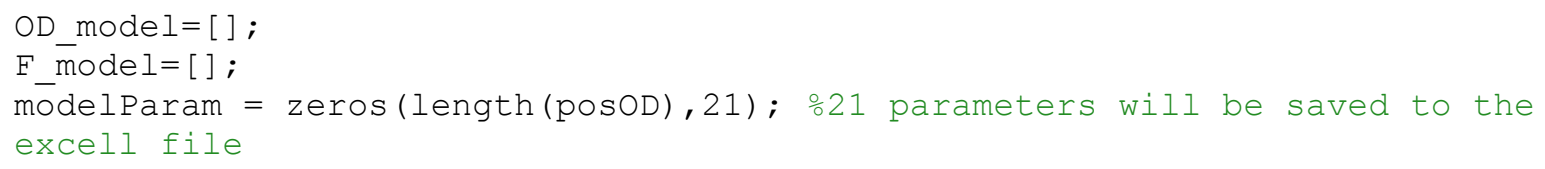




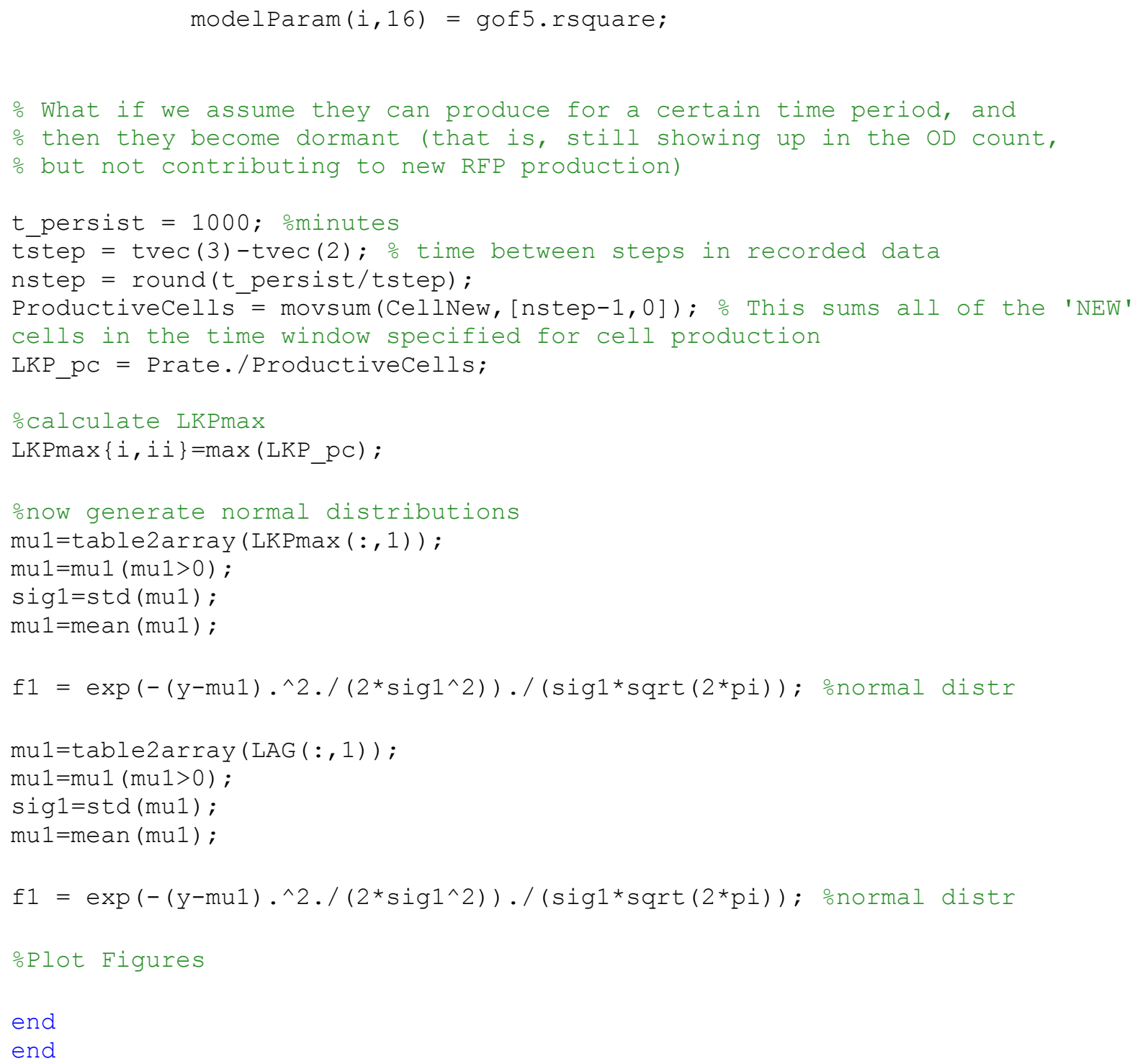

\section{5. qPCR and RT-qPCR data processing.}

All qPCR and RT-qPCR data was acquired on ABI StepOne Plus thermocycler. Both probes were cycled simultaneously with a 60 second initial denaturation at $95 \mathrm{C}$, and then 40 cycles of 15 second denaturation at $95 \mathrm{C}$ followed by 30 second extension step at $60 \mathrm{C}$. RT-qPCR samples were subjected to an additional reverse transcription step at $55 \mathrm{C}$ for 10 minutes.

Fluorescence data that was collected by the thermocycler was exported into an excel file. That data was then sorted using custom matlab code (qPCR_data_analyzer.m). Organized data was then fit with a 5 parameter logistics curve (qPCR2mat_V2.m). Raw data was normalized by subtracting fluorescence detected at the first step from fluorescence at each step of amplification. Normalized data was then converted to the log scale. Threshold values were then set manually. Linear standard curves were fitted through the $\mathrm{C}_{\mathrm{T}}$ vs DNA or cell concentration data, in order to obtain the efficiency values (plotqPCR.m). 
When the efficiency values were confirmed to be in the same range, $C_{T}$ values of the reference and unknowns were used to calculate relative differences in concentration using $2^{-\Delta C}{ }_{T}$ method (Supplement figure 5). This series of 3 programs was used to process qPCR data.

In order to acquire samples for qPCR experiments, $B$. subtilis 168 cells with either a theta replicating or integrating plasmid were grown in a 6 well plate and harvested at different stages of growth (approximately every 2 hours, which corresponded to: pre-log, early log, mid log, late log and stationary growth phases. Time points $1,2,3,4$, and 5 respectively) according to the methods section.

This qPCR experiment was performed twice for $B$. subtilis 168 cells with a theta replicating plasmid, and twice for B. subtilis 168 with the integrating plasmid (Supplement Figures 6-9).

Two duplicate cell cultures of $B$. subtilis 168 were used to extract cell supernatants for this qPCR experiment, resulting in two supernatant samples. Each cell supernatant sample was diluted 10 fold 4 times $(1 \mathrm{X}, 0.1 \mathrm{X}, 0.001 \mathrm{X}$ and $0.0001 \mathrm{X})$ as described in the method section. This resulted in 8 curves corresponding to $B$. subtilis 168 supernatant samples qPCR amplified with a riboswitch specific probe (blue; Supplement Figure 6A), and 8 more curves corresponding to same $B$. subtilis 168 supernatant samples amplified with a reference probe (red; Supplement Figure 6A). As these duplicate cell cultures were propagating, cell samples were pulled from each culture 5 times every 2 hours during the growth period (Times 1-5; pre-log, early log, mid log, late log, and stationary growth phases). Raw linear fluorescence data acquired with the $A B I$ thermocycler was baseline normalized for each fraction of cells that were harvested at different stages of growth (Times 1-5; Supplement Figure 6A, 6D, 6G, 6J, 6M). Normalized raw linear fluorescence data was converted to the logarithmic scale in order to set threshold values (Supplement Figure 6B, 6E, 6H, 6K, 6N). Optimal threshold values were set to 2 for the reference probe (dashed red line, Supplement Figure $6 \mathrm{~B}, 6 \mathrm{E}, 6 \mathrm{H}, 6 \mathrm{~K}, 6 \mathrm{~N}$ ) and 3 for the riboswitch specific probe (dashed blue line, Supplement Figure $6 \mathrm{~B}, 6 \mathrm{E}, 6 \mathrm{H}, 6 \mathrm{~K}, 6 \mathrm{~N}$ ). Then, $\mathrm{C}_{\mathrm{T}}$ values were calculated where the threshold line intersected the baseline normalized logarithmic data from each sample. This resulted in 8 data points for $B$. subtilis supernatant sample qPCR amplified with a riboswitch specific probe (crosses, Supplement Figure 6C, 6F, 6I, 6L, 60), and 8 data points for B. subtilis supernatant sample qPCR amplified with a reference probe (circles, Supplement Figure 6C, 6F, 6I, 6L, 60). Data points from the reference and riboswitch specific probes were fit with a linear functions, red and blue respectively.

As a result, Supplement Figures 6 and 7 correspond to trials 1 and 2 of the qPCR experiment that was performed on duplicate cultures of $B$. subtilis 168 cells that carried a theta replicating plasmid, in order to calculate the copy number of the plasmid. Supplement Figures 8 and 9 correspond to trials 1 and 2 of the qPCR experiment with $B$. subtilis 168 that carried an integrating plasmid. 


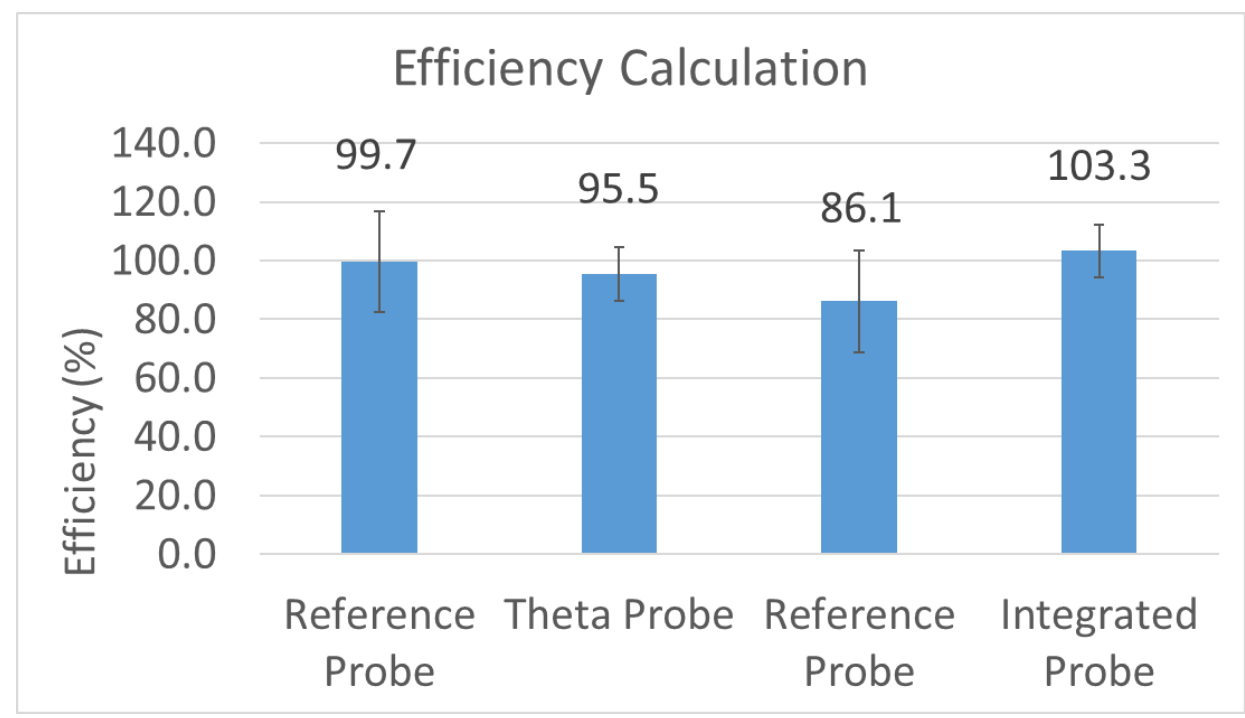

Supplement Figure 5 - qPCR amplification efficiency data. Cell supernatant samples were used as templates in qPCR reactions with either a reference or riboswitch DNA probes.

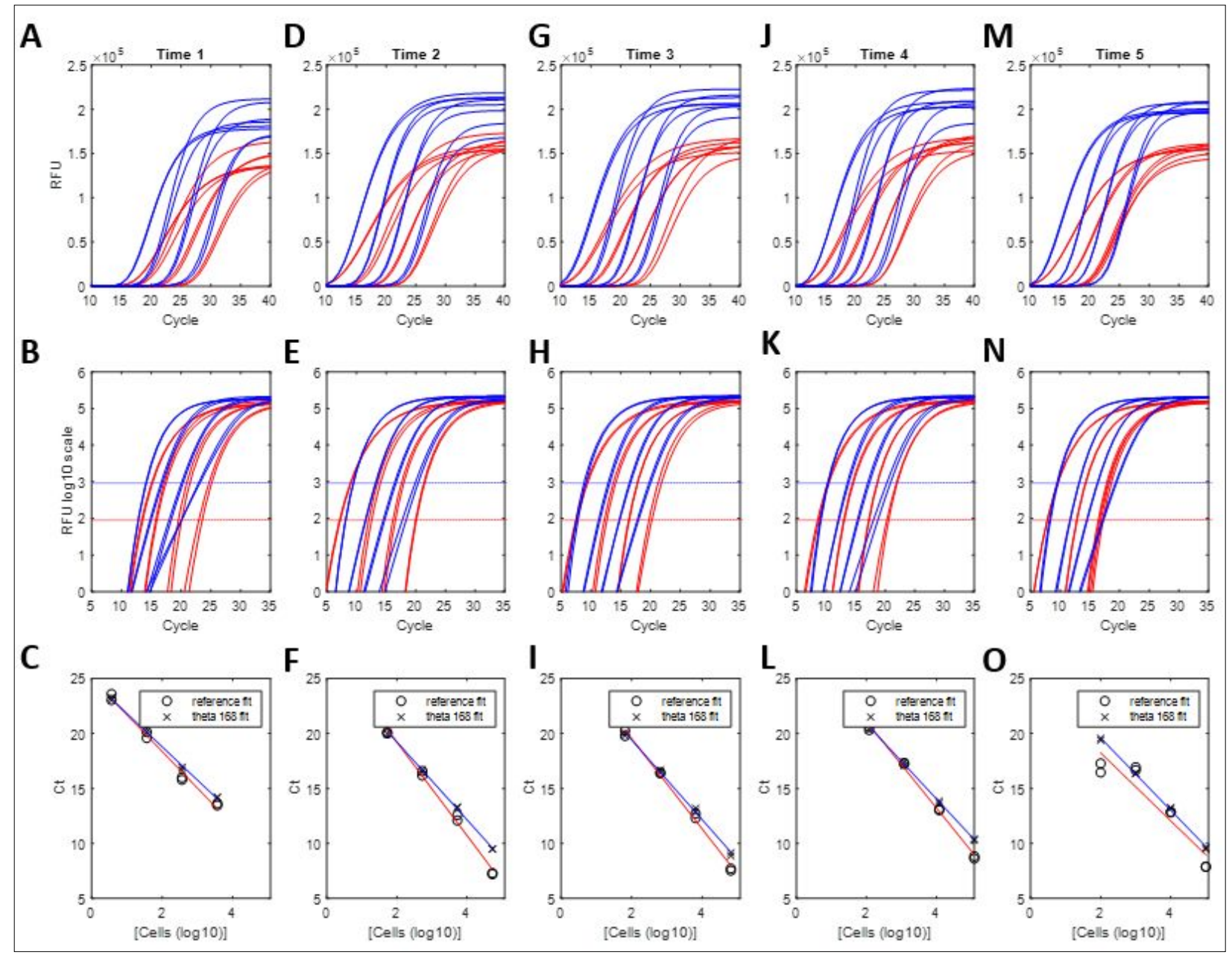

Supplement figure 6 - Trial 1 of the qPCR data from B. subtilis 168 that carried a theta replicating plasmid. (A, D, G, J, and M) correspond to baseline normalized linear fluorescence data that was acquired with an ABI StepOne Plus thermocycler. Blue 
curves (8) correspond to data acquired on the cell supernatant that was qPCR amplified with the riboswitch specific probe, and red curves (8) to reference probe. Supernatant samples were acquired from 2 separate cell cultures of $B$. subtilis 168 . Each supernatant sample was diluted 10 fold, 3 times $(1 X, 0.1 X, 0.01 X$, and $0.001 X)$. (B, E, $\mathbf{H}, K$ and $\mathbf{N})$ correspond to baseline normalized linear fluorescence data converted to the logarithmic scale. $(C, F, I, L$, and $O)$ correspond to $C_{T}$ values acquired from the logarithmic data. Circles and crosses represent the $C_{T}$ values from the reference and riboswitch specific probes respectively. Data from each probe was fit with a linear function (red-reference, blues - riboswitch sample).

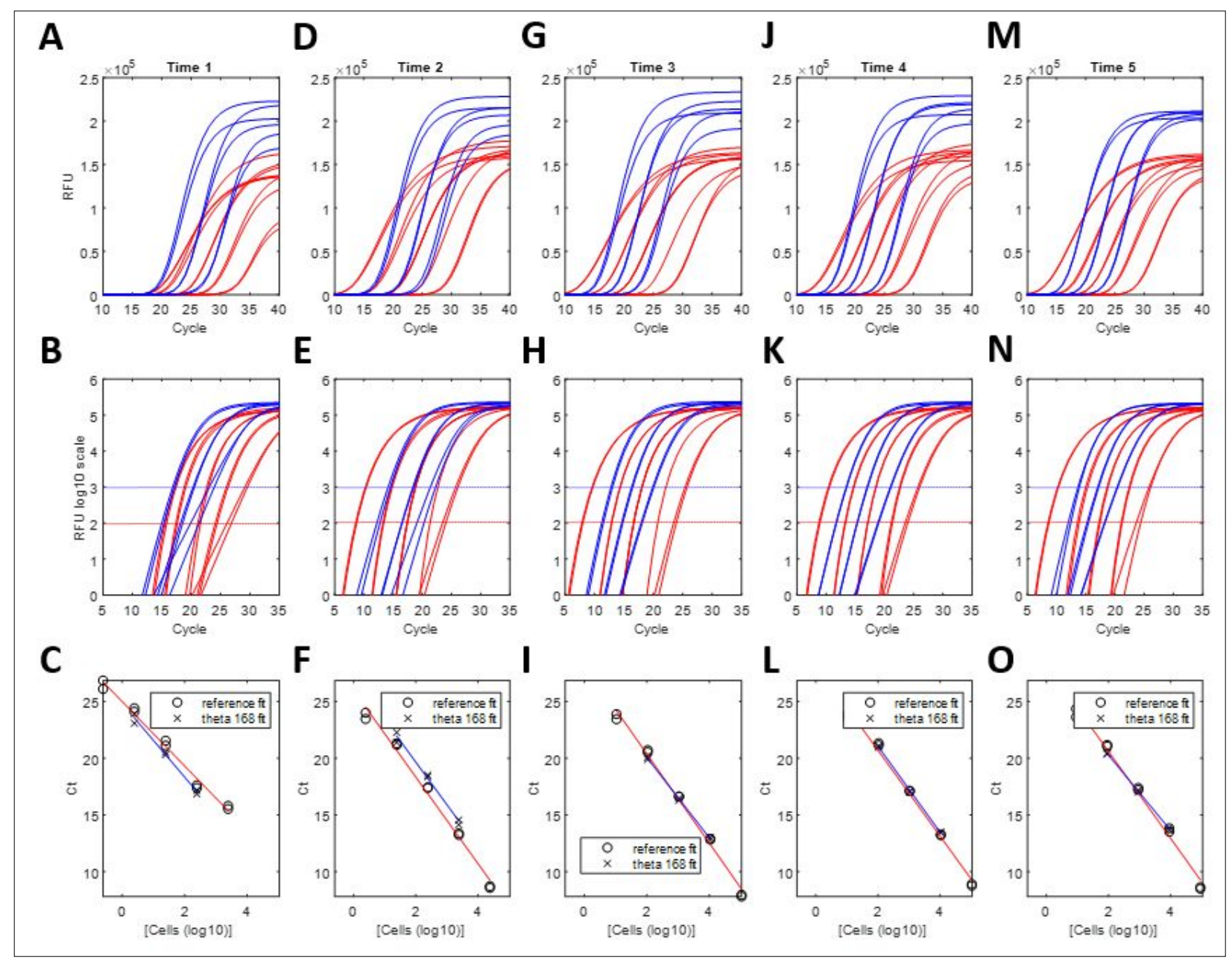

Supplement figure 7-Trial 2 of the qPCR data from $B$. subtilis 168 that carried a theta replicating plasmid. (A, D, G, J, and M) correspond to baseline normalized linear fluorescence data that was acquired with an ABI StepOne Plus thermocycler. Blue curves (8) correspond to data acquired on the cell supernatant that was qPCR amplified with the riboswitch specific probe, and red curves (8) to reference probe. Supernatant samples were acquired from 2 separate cell cultures of $B$. subtilis 168 . Each supernatant sample was diluted 10 fold, 3 times $(1 X, 0.1 X, 0.01 X$, and $0.001 X)$. (B, E, $\mathrm{H}, \mathrm{K}$ and $\mathrm{N})$ correspond to baseline normalized linear fluorescence data converted to the logarithmic scale. $(C, F, I, L$, and $O)$ correspond to $C_{T}$ values acquired from the logarithmic data. Circles and crosses represent the $C_{T}$ values from the reference and riboswitch specific probes respectively. Data from each probe was fit with a linear function (red-reference, blues - riboswitch sample). 


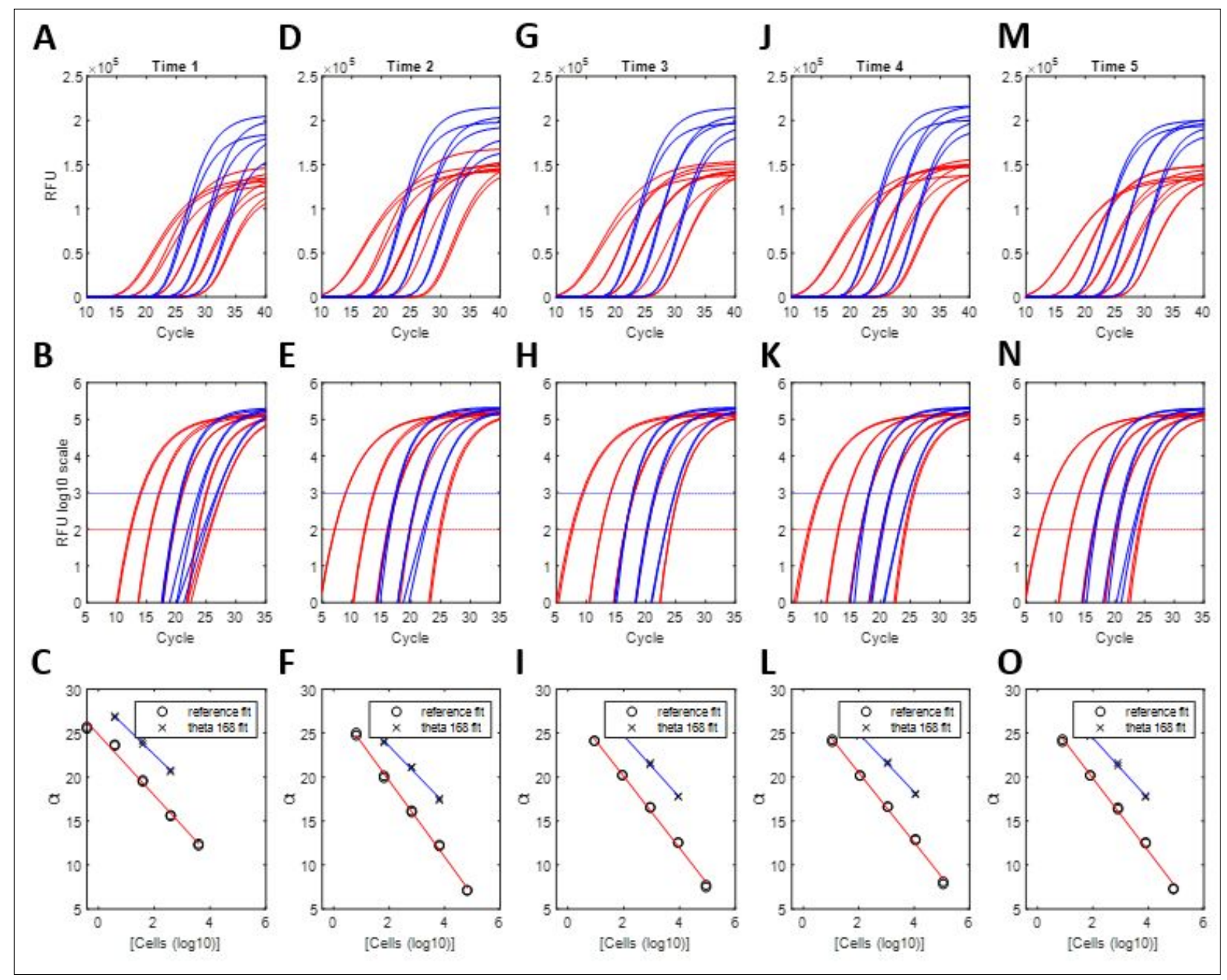

Supplement figure 8 - Trial 1 of the qPCR data from B. subtilis 168 that carried an integrating plasmid. $(A, D, G, J$, and $M)$ correspond to baseline normalized linear fluorescence data that was acquired with an ABI StepOne Plus thermocycler. Blue curves (8) correspond to data acquired on the cell supernatant that was qPCR amplified with the riboswitch specific probe, and red curves (8) to reference probe. Supernatant samples were acquired from 2 separate cell cultures of $B$. subtilis 168 . Each supernatant sample was diluted 10 fold, 3 times $(1 X, 0.1 X, 0.01 X$, and $0.001 X)$. (B, E, $\mathbf{H}, \mathbf{K}$ and $\mathbf{N})$ correspond to baseline normalized linear fluorescence data converted to the logarithmic scale. $(C, F, I, L$, and $O)$ correspond to $C_{T}$ values acquired from the logarithmic data. Circles and crosses represent the $C_{T}$ values from the reference and riboswitch specific probes respectively. Data from each probe was fit with a linear function (red-reference, blues - riboswitch sample). 


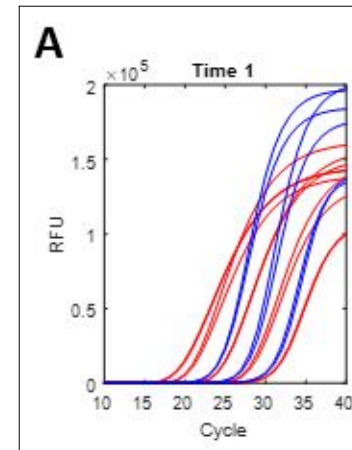

B
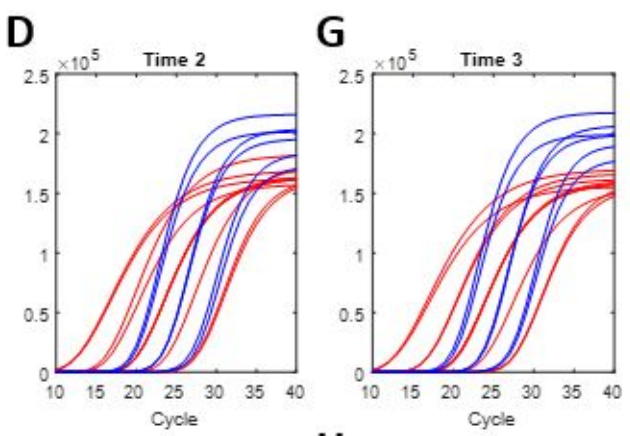

$\mathbf{E}$

H

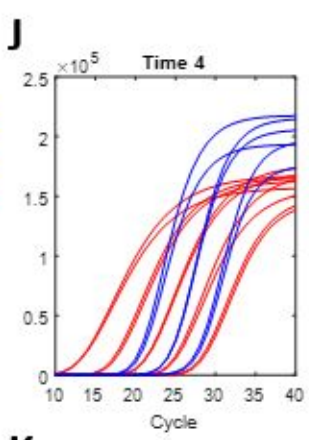

M
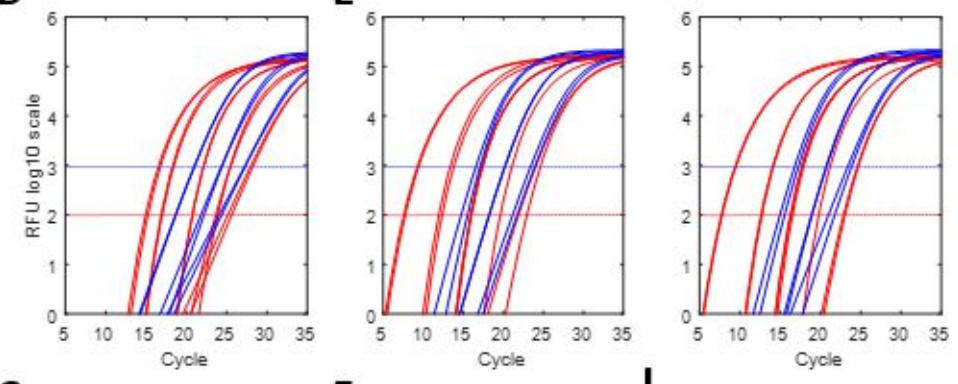

K

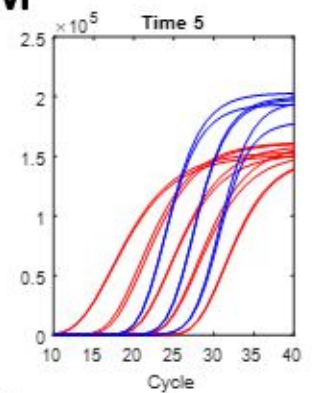

$\mathbf{N}$
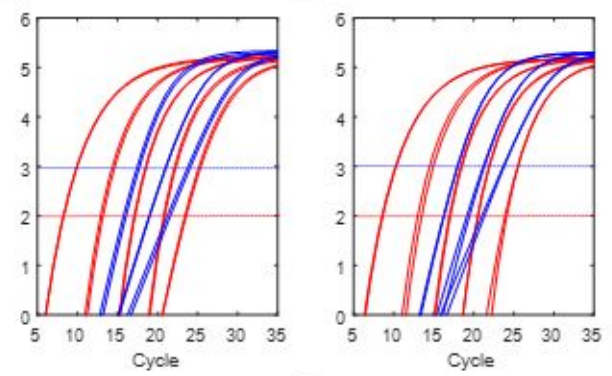

C
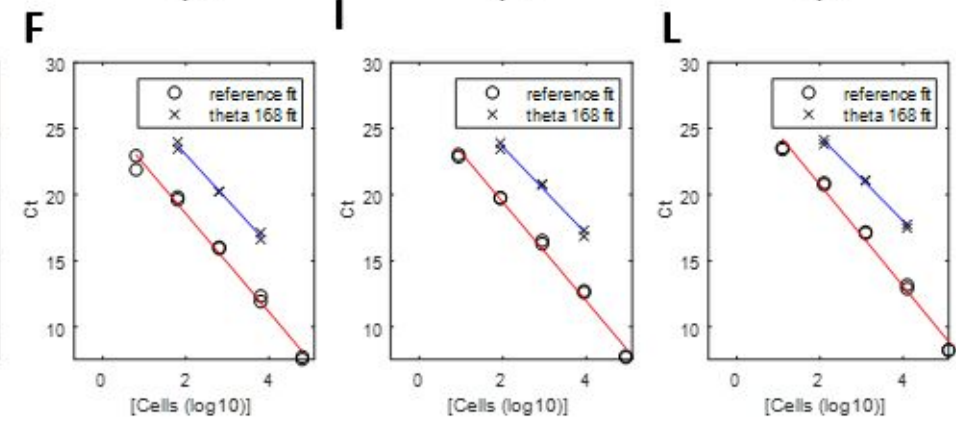

$\mathbf{O}$
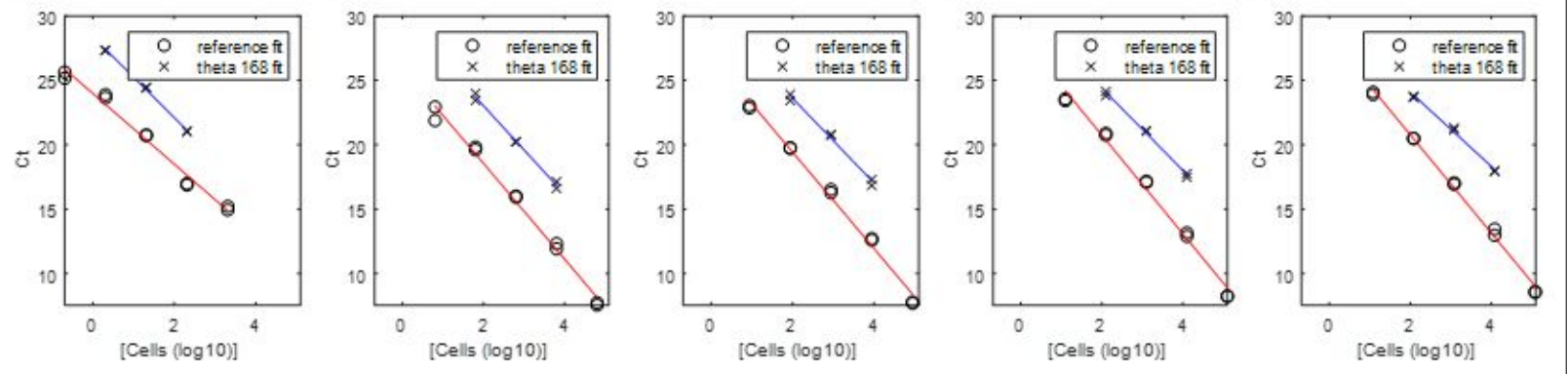

Supplement figure 9 - Trial 2 of the qPCR data from B. subtilis 168 that carried an integrating plasmid. (A, D, G, J, and M) correspond to baseline normalized linear fluorescence data that was acquired with an ABI StepOne Plus thermocycler. Blue curves (8) correspond to data acquired on the cell supernatant that was qPCR amplified with the riboswitch specific probe, and red curves (8) to reference probe. Supernatant samples were acquired from 2 separate cell cultures of $B$. subtilis 168 . Each supernatant sample was diluted $10 \mathrm{fold}, 3$ times $(1 X, 0.1 X, 0.01 X$, and $0.001 X)$. (B, E, $\mathrm{H}, \mathrm{K}$ and $\mathrm{N})$ correspond to baseline normalized linear fluorescence data converted to the logarithmic scale. $(C, F, I, L$, and $O)$ correspond to $C_{T}$ values acquired from the logarithmic data. Circles and crosses represent the $C_{T}$ values from the reference and riboswitch specific probes respectively. Data from each probe was fit with a linear function (red-reference, blues - riboswitch sample).

In order to calculate the relative copy number of the riboswitch genetic circuit in B. subtilis cells with either a theta replicating or integrating plasmid, $2^{-\Delta C}$ Tethod was used. Relative copy number was calculated for each of the cell fractions harvested at different stages of growth (pre-log, early log, mid $\log$, late log and stationary growth phases. Time points 1, 2, 3, 4, and 5 respectively). For each time point, the error bars overlapped greatly, and it was concluded that there are no growth phase dependent fluctuations in the relative copy number. Specifically, the relative copy number of both a theta replicating and integrating plasmids stayed constant regardless of whether cells were in the early log or stationary growth phases (Supplement Figure 10). Since there were no growth phase dependent trends in the relative copy number, all time points were averaged to get the overall ratio of relative copy numbers of either theta replicating or integrating plasmids in B. subtilis 168 cells (Supplement Figure 11). 
It was concluded that, since the reference, $16 \mathrm{~s}$ rDNA, is present in $B$. subtilis cells in 10 copies, the 1:1 ratio of the theta plasmid was converted to 10 copies of the expression cassette. Conversely, since about a 1:10 ratio of reference 16 s rDNA to integrated DNA was observed, the copy number of expression cassette integration was estimated to be equal to 1 .

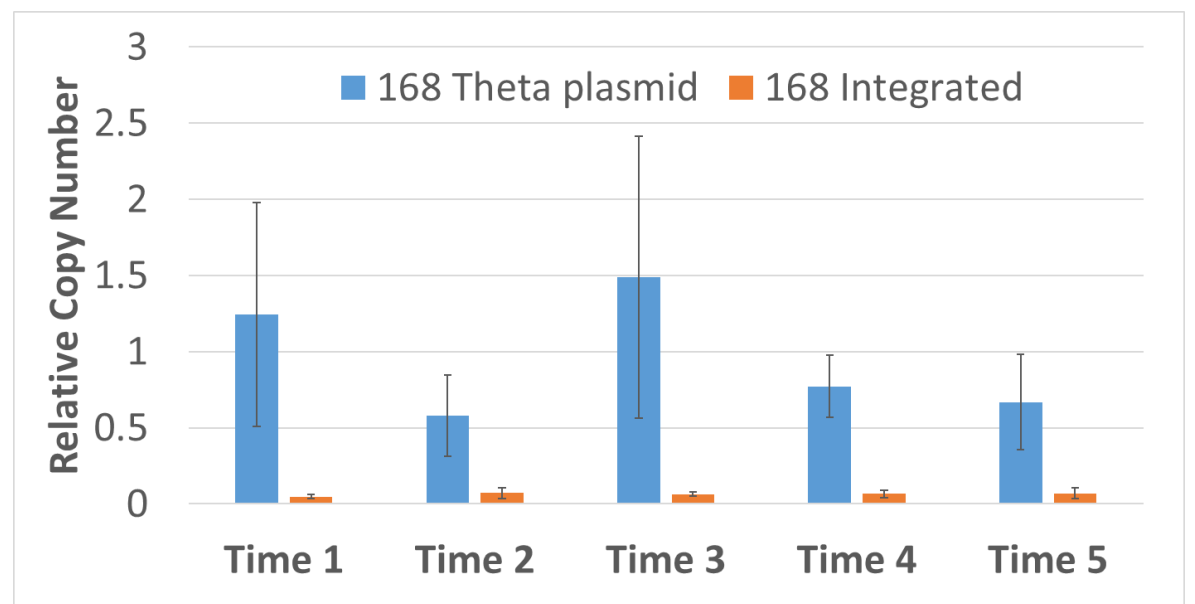

Supplement figure 10 - Average relative copy number values of wild type cells carrying either a theta plasmid or integrated DNA harvested at different growth phases (pre-log, early log, mid log, late log and stationary growth phases. Time points 1, 2, 3, 4, and 5 respectively).

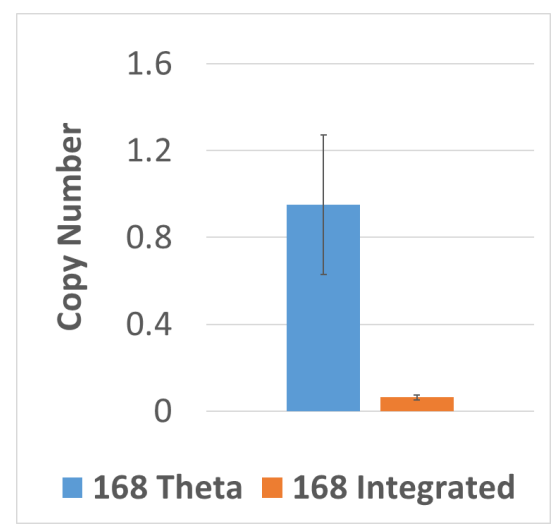

Supplement figure 11- Average, across all growth phases, relative copy number values of wild type cells carrying either a theta plasmid or integrated DNA harvested at different times during the growth.

In order to perform the RT-qPCR experiment, the supernatants of B. subtilis 168 cells used in the first trial of the qPCR experiment were stored at -80C. These supernatants were then thawed, and RNA was extracted according to the protocol in the method section. RNA concentrations were estimated using a ThermoFisher nanoDrop (Supplement Tables 3 - 4).

RT-qPCR data processing workflow was similar to that of qPCR data. Specifically, two duplicate cell cultures of $B$. subtilis 168 carrying a theta replicating and integrating plasmid (4 cultures in total) from trial 1 of the qPCR experiment were used. The supernatants from these duplicate cultures were then diluted to $0.1 \mathrm{X}, 0.001 \mathrm{X}$ and $0.00001 \mathrm{X}$. This resulted in $6 \mathrm{RT}$-qPCR amplification experiments for the supernatant with the riboswitch that was located in the genome (3 dilutions of duplicates; Supplement Figure 12A, green curves), and 6 more curves for the supernatant with the riboswitch that was located on the theta replicating plasmid (Supplement Figure 12A, blue curves). 
In order to quantify riboswitch specific RNA relative copy number, absolute quantification method was used. In order to acquire the standard solution of the DNA that contained the theophylline riboswitch, pHT plasmid was propagated overnight in Top10 E. coli cells and miniprepped. A serial dilution of extracted DNA was performed, and an 8 point standard curve was prepared. Each dilution of the standard solution was used twice in the RT-qPCR experiment, resulting in 16 samples (Supplement Figure 12A, red curves).

This RT-qPCR experiment was performed on each fractions of the cells harvested at 5 different time points, to get a temporal resolution of the fluctuations of the RNA concentration in B. subtilis 168 cells during various stages of growth. First, baseline normalized linear fluorescence data was plotted (Supplement Figure 12A, 12D, 12G, 12J, 12M). Then, this baseline normalized linear fluorescence data was converted to the logarithmic scale (Supplement Figure 12B, 12E, 12H, 12K, and 12N). The threshold value was set at 3 since it was assumed that the length and structure of the RNA transcribed from the genome and from the plasmid was similar, and therefore would not introduce variability in probe binding. Points where $C_{T}$ values were reached for each individual sample of the standard curve were plotted and fit with a linear function (Supplement Figure 12C, 12F, 12I, 12L, 12O). This standard curve was used to calculate the amount of riboswitch RNA that was found in each sample. The total concentration of the RNA in each RT-qPCR reaction was used to normalize the copy number of riboswitch specific RNA that was acquired through this RT-qPCR experiment (Supplement Table 5). Finally, the normalized copy number of the riboswitch specific RNA was normalized again by the absolute copy number of DNA that was determined with qPCR experiments (Supplement Table 6). These RT-qPCR results were depicted on figures $6 A$ and $6 B$ and discussed in the main manuscript.

Supplement table 2 RNA concentration after RNA purification

\begin{tabular}{|c|c|c|c|c|c|c|c|}
\hline & \multicolumn{2}{|c|}{ Theta } & \multicolumn{4}{|c|}{ Integrating } & \\
\hline & Trial 1 & Trial 2 & AVG & Trial 1 & Trial 2 & & \\
\hline Time 1 & 38.298 & 46.915 & 42.6065 & 24.065 & 34.194 & 29.1295 & $\mathrm{ng} / \mathrm{ul}$ \\
\hline Time 2 & 52.504 & 58.531 & 55.5175 & 31.84 & 36.602 & 34.221 & $\mathrm{ng} / \mathrm{ul}$ \\
\hline Time 3 & 688.631 & 786.852 & 737.7415 & 32.277 & 31.107 & 31.692 & g/ul \\
\hline Time 4 & 58.19 & 58.58 & 58.385 & 56.29 & 57.222 & 56.756 & $\mathrm{ng} / \mathrm{ul}$ \\
\hline Time 5 & 166.547 & 185.935 & 176.241 & 218.629 & 219.712 & 219.1705 & $\mathrm{ng} / \mathrm{ul}$ \\
\hline
\end{tabular}

Supplement table 3 RNA concentration in RT-qPCR reactions (dilution replicates)

\begin{tabular}{|c|c|c|c|c|c|c|}
\hline & & Theta & & & Integrating & \\
\hline & $0.1 X$ & $0.001 X$ & $0.00001 X$ & $0.1 X$ & $0.001 X$ & $0.00001 X$ \\
\hline Time 1 & 4.26065 & 0.042607 & 0.000426 & 2.91295 & 0.02913 & 0.000291 \\
\hline Time 2 & 5.55175 & 0.055518 & 0.000555 & 3.4221 & 0.034221 & 0.000342 \\
\hline Time 3 & 73.77415 & 0.737742 & 0.007377 & 3.1692 & 0.031692 & 0.000317 \\
\hline Time 4 & 5.8385 & 0.058385 & 0.000584 & 5.6756 & 0.056756 & 0.000568 \\
\hline Time 5 & 17.6241 & 0.176241 & 0.001762 & 21.91705 & 0.219171 & 0.002192 \\
\hline
\end{tabular}


Supplement table 4 Theophylline RNA normalized by total RNA in reaction (figure 5A)

\begin{tabular}{|ll|rr|}
\hline \multicolumn{2}{|c|}{ Theophylline RNA (copies) / RNA total (ng) } \\
\multicolumn{2}{|c|}{ Theta } & \multicolumn{2}{|c|}{ Integrating } \\
AVG & STDEV & \multicolumn{1}{|l|}{ AVG } & \multicolumn{1}{l|}{ STDEV } \\
10838033 & 129778.04 & 1342857.6 & 44901.413 \\
3691818.1 & 449778.73 & 346364.57 & 22150.31 \\
805934.65 & 46094.536 & 128756.53 & 14564.752 \\
1161384.5 & 238352.27 & 64019.88 & 745.32271 \\
646788.43 & 73095.961 & 17712.588 & 3300.6171 \\
\hline
\end{tabular}

Supplement table 5 Riboswitch RNA normalized by total RNA in RT-qPCR reaction and DNA copy number (figure 5B)

\begin{tabular}{|lrl|lll|}
\hline \multicolumn{3}{|c|}{ Theta } & \multicolumn{3}{c|}{$\begin{array}{l}\text { Integrating } \\
\text { Error }\end{array}$} \\
Average & Error High & Error Low & Average & High & Low \\
1205297.3 & 2494822 & 788080.41 & 2581425.6 & 5026655 & 1698184 \\
410566.96 & 942079.19 & 238599.81 & 665829.62 & 1334812 & 424186.5 \\
89627.964 & 193809.06 & 55920.884 & 247513.51 & 519129.5 & 149403.1 \\
129157.52 & 318394.79 & 67931.103 & 123067.82 & 234588.5 & 82785.43 \\
71929.319 & 163750.39 & 42221.238 & 34049.572 & 76112.74 & 18855.94 \\
\hline
\end{tabular}




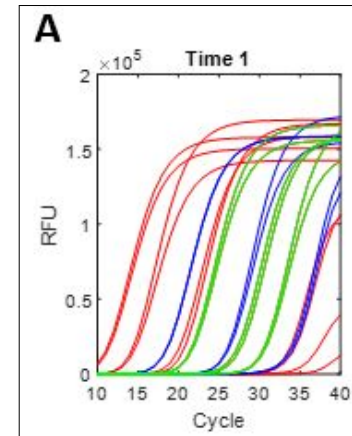

B
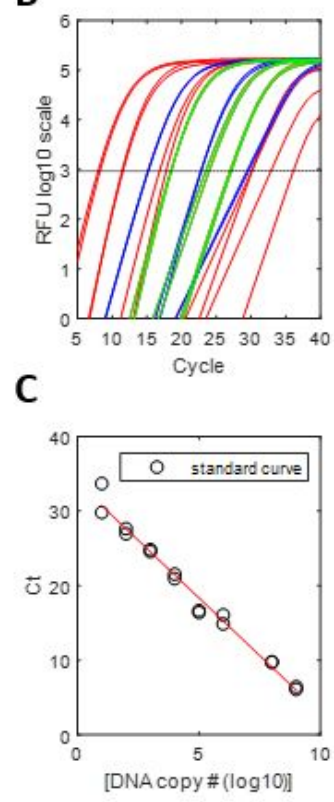

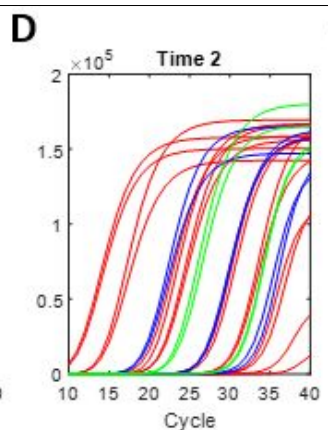

E
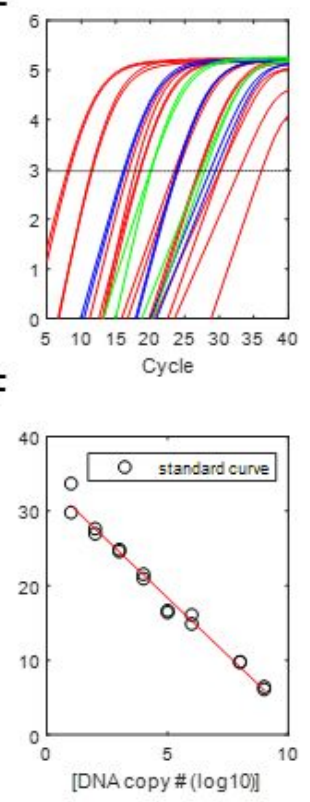

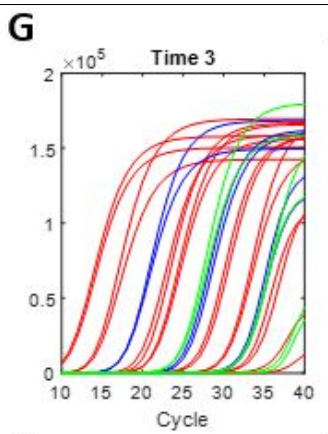

$\mathbf{H}$

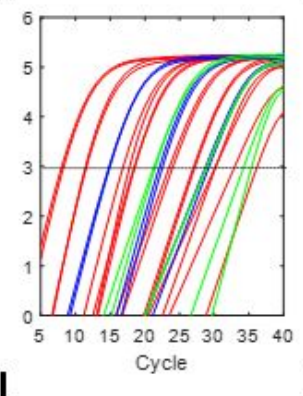

।

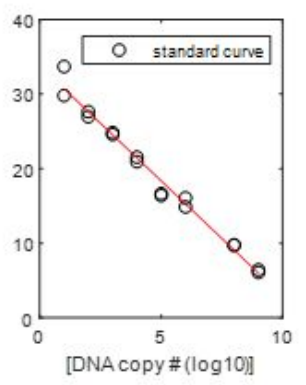

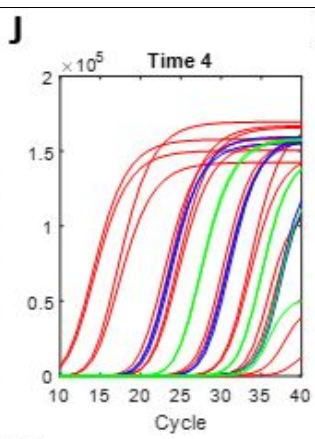

K
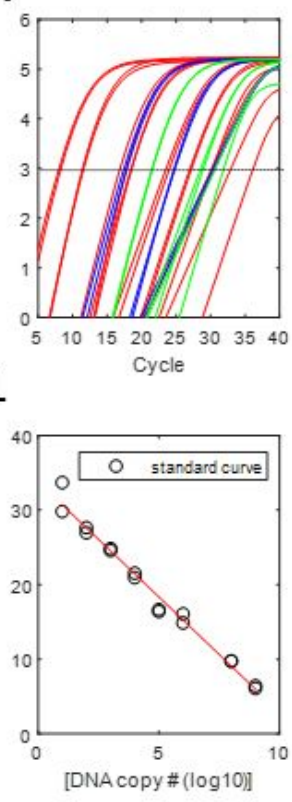

M

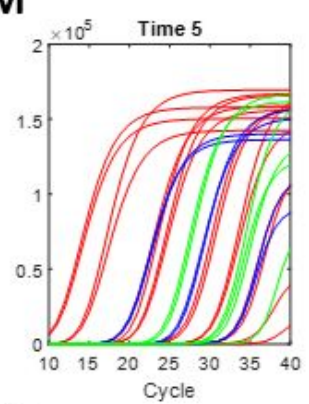

N

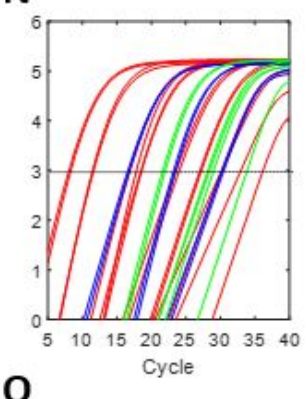

$\mathbf{O}$

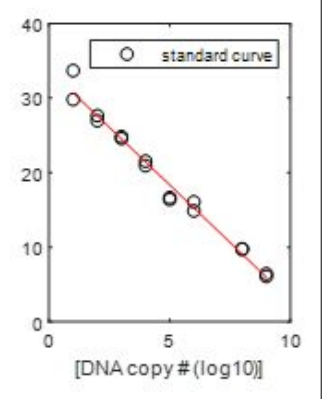

Supplement figure $12-R T-q P C R$ data and processing workflow from B. subtilis 168 that carried an integrating plasmid (green curves), a theta replicating plasmid (blue curves), and purified DNA (red curves). (A, D, G, J, and $M$ ) correspond to baseline normalized linear fluorescence data that was acquired with an ABI StepOne Plus thermocycler. Blue curves (6: 3 dilutions of duplicates) correspond to data acquired on the cell supernatant of B. subtilis 168 cells with a theta replicating plasmid that was $R T$-qPCR amplified with the riboswitch specific probe, green curves (6: 3 dilutions of duplicates) B. subtilis 168 supernatant with integrating plasmid (riboswitch integrated in the chromosome), and red curves (16: 8 standard points in duplicates) corresponding to the purified standard DNA. (B, E, $\boldsymbol{H}, \boldsymbol{K}$ and $N)$ correspond to baseline normalized linear fluorescence data converted to the logarithmic scale. $(C, F, I, L$, and $O)$ correspond to $C_{T}$ values acquired from the logarithmic data of the standard DNA samples.

\section{Below is an example of the custom code that was used to process qPCR and R-qPCR data:}

\%coded on 11/15/2018 by DDT

omodified for experiment 6 on 01/24/2019 by DDT

othe code fluorescence data for all 4 channels from all wells and organizes it per cycle.

ocode requires raw data output from OneStep software in a separate .xlsx file oColumns grouped in 4 per each well (B, G, Y, R). Order of wells dictated by the well matrix.

function qPCR_data_analyzer 


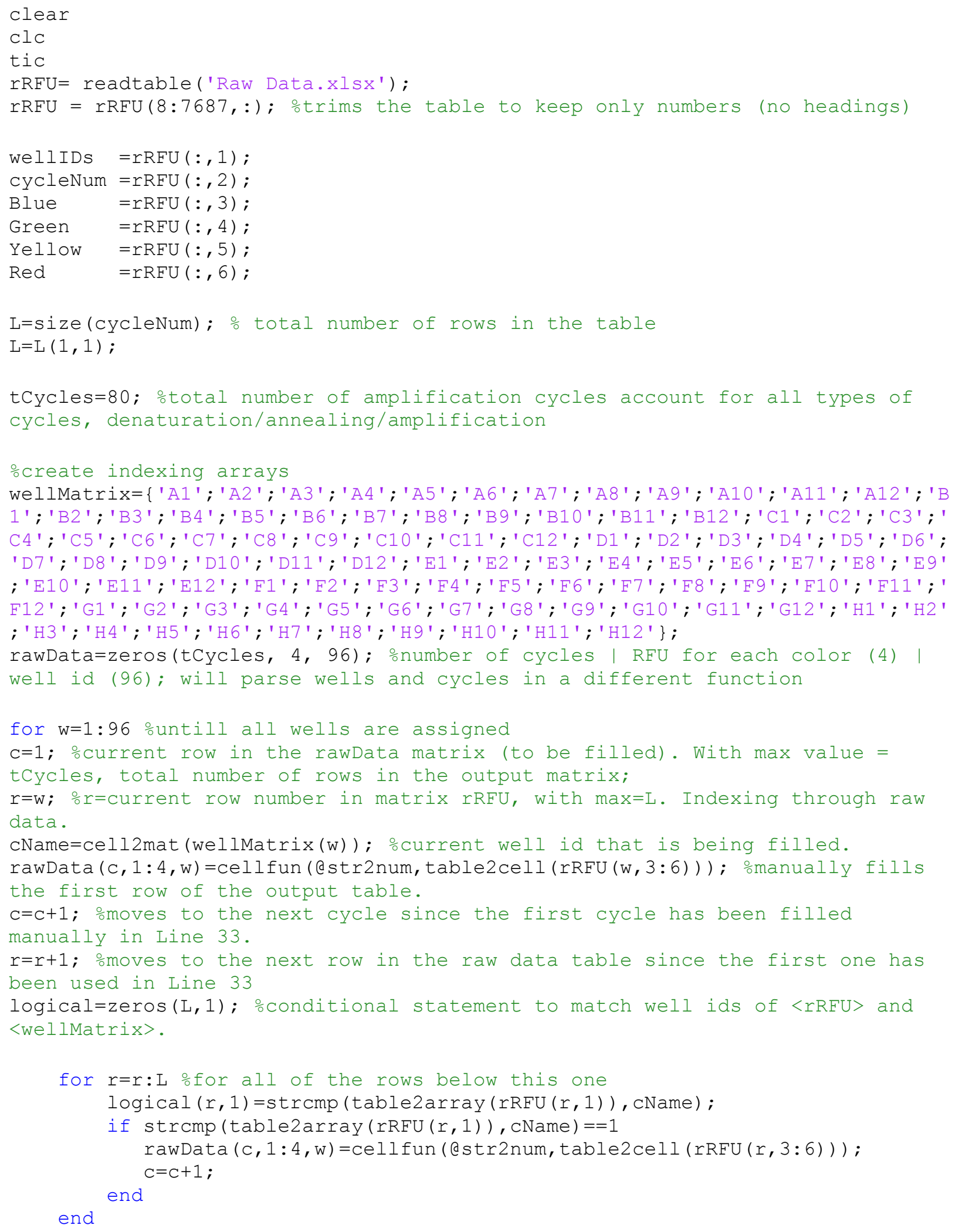

end 
oThe data will be organized in 80 rows (one for each cycle)

oFour columns for each well, the order of wells is dictated by the well

matrix.

csvwrite ('Organized data.csv', rawData);

toc

end

coded on 11/15/2018 by DDT

V2 modified on 01/06/2018

\%ㅇodification objective is to automatically fit every well and generate othis matlab file must be located in the same folder as the data file

$\frac{0}{0}$

oinput data requirements: you need (2) files.

oqPCR data from AIB OnestepPlus exports data in the .xls file. Raw data

ofrom that file will be parsed and organized by the function

o"qPCR_data_analyzer.m". The output of that function is "data.csv" (see

o"qPCR_data_analyzer.m" for details on data structure in data.csv). After

othat, you will need legends that were assigned to this data in the

\%OneStepplus software. Onestepplus takes data for all channels and all

owells. To only keep necessary data this program will crossreference raw

odata from "data.csv" and the legened.

\%(1) import data.csv using $[\sim, \sim,<$ name> $]=x l s r e a d(' d a t a . c s v ')$;

\%(2) import the legend from the original OnestepPlus output file using

\% $[\sim, \sim,<$ name $>$ ] =xlsread ('<name of the OnestepPlus output file>', 'Sample

ㅇetup');

osystem output is a structured array with columns: [well] [type] [dilution]

\% [RFUprobe ] [RFUrox]

응 \{

EXAMPLES OF USAGE:

$[\sim, \sim$, data $]=$ xlsread ('organized data.CSV'); ooutput from qPCR_data_analyzer

$[\sim, \sim$, legend] = xlsread('Experiment 4','Sample Setup');

qPCRdata=qPCR2mat (data, legend);

응

function [qPCRdata] =qPCR2mat_V2 (data, legend, $C t)$

tic

owill need this at some point for crossreference

wellMatrix=\{'A1'; 'A2'; 'A3'; 'A4'; 'A5';'A6';'A7'; 'A8'; 'A9'; 'A10'; 'A11';'A12'; 'B

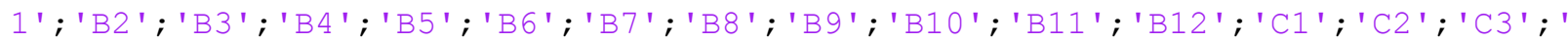
C4';'C5';'C6';'C7';'C8';'C9';'C10';'C11';'C12';'D1';'D2';'D3';'D4';'D5'; 'D6'; 'D7';'D8';'D9'; 'D10'; 'D11';'D12';'E1';'E2';'E3';'E4';'E5';'E6';'E7';'E8'; 'E9' ;'E10';'E11';'E12';'E1';'F2';'F3';'F4';'F5';'F6';'F7';'F8';'F9';'F10';'F11';' F12';'G1';'G2';'G3';'G4';'G5';'G6';'G7';'G8'; 'G9';'G10';'G11';'G12';'H1'; 'H2' ;'H3'; 'H4'; 'H5'; 'H6' ; 'H7', 'H8'; 'H9'; 'H1O'; 'H11' ; 'H12'\};

oinput RFU data

input $=$ data ;

oParse the legend file to remove headers

vLength=length (legend); 
$\mathrm{N}=$ find (strcmp (legend, 'Well')) ;

legend=cell2table (legend (N+1:vLength, 1:12));

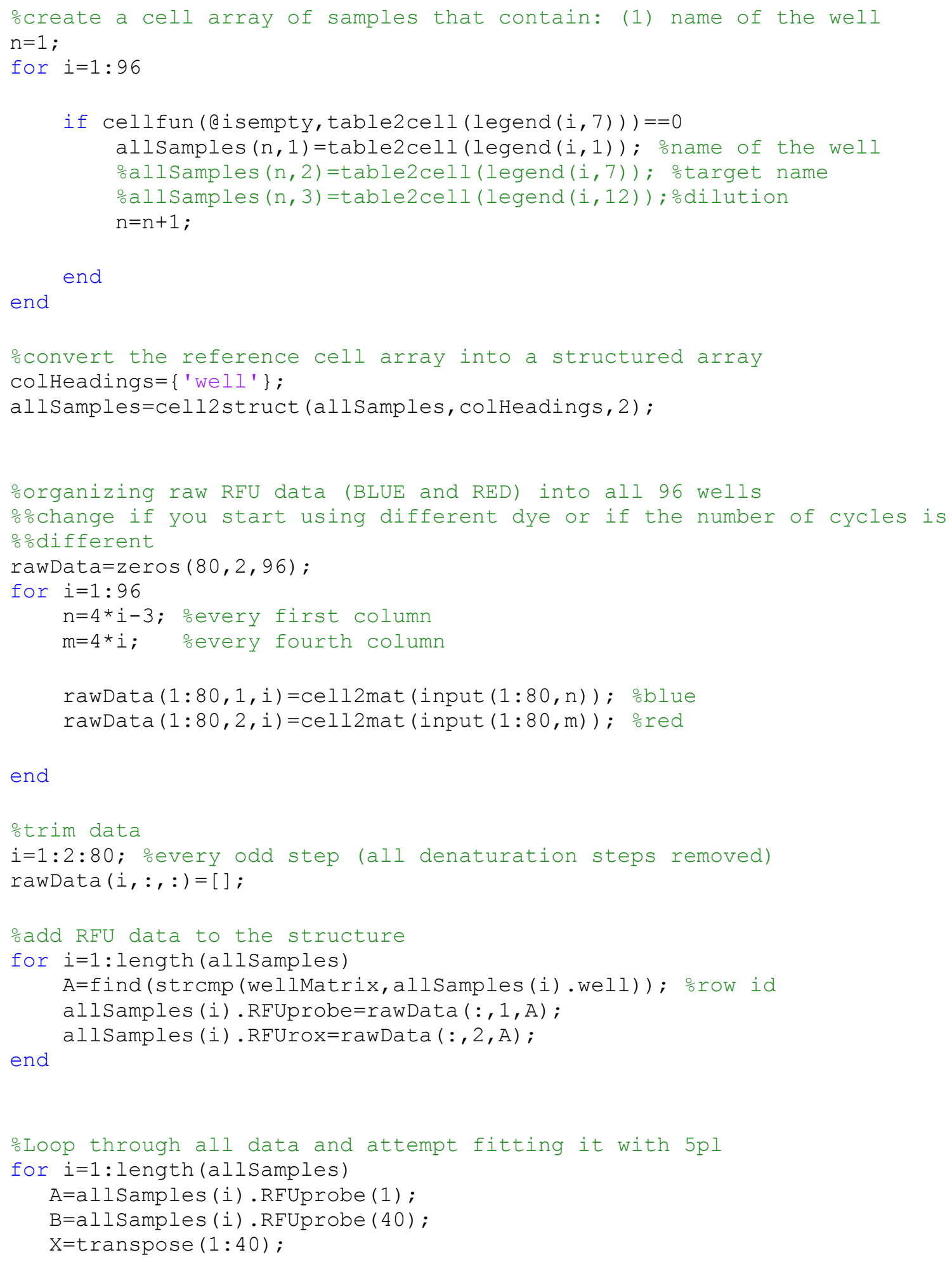


if $\mathrm{A}>0$ \&\& $\mathrm{B}>0$ \&\& $\mathrm{B}>\mathrm{A}$ 의 there is signal from the well ( negative RFU and final RFU > initial RFU)

y=allsamples (i). RFUprobe;

[xData, yData $]=$ prepareCurveData $(\mathrm{X}, \mathrm{y})$;

ocustomized guess parameters for upper and lower bounds:

$\mathrm{cMin}=\mathrm{y}(1)-\mathrm{y}(1) * 0.05 ; \% 5$ percent below min

$\mathrm{cMax}=\mathrm{y}(1)+\mathrm{y}(1) * 0.05 ; \% 5$ percent above min

$\mathrm{dMin}=\mathrm{y}(40)-\mathrm{y}(40) * 0.05$;

$\mathrm{dMax}=\mathrm{y}(40)+\mathrm{y}(40) * 0.05$;

$\operatorname{try}$

$\mathrm{ft}=$ fittype $\left({ }^{\prime} \mathrm{c}+(\mathrm{d}-\mathrm{c}) /(1+\exp (\mathrm{b} *(\mathrm{x}-\mathrm{e})))^{\wedge} \mathrm{f}\right.$ ', 'independent', 'x',

'dependent', 'y');

opts = fitoptions ( 'Method', 'NonlinearLeastSquares' );

opts.Display = 'Off';

opts. Lower $=\left[\begin{array}{lll}-1 & \mathrm{cMin} \\ \mathrm{dMin} & - \text { Inf } 0\end{array}\right]$;

oopts.StartPoint $=[0.8166758356677260 .64689910993646$

$0.9133758561390190 .632359246225410 .0975404049994095]$;

opts.StartPoint $=\left[\begin{array}{llll}0.816675835667726 & 0.8 & 0.913375856139019\end{array}\right.$

$0.632359246225410 .0975404049994095]$;

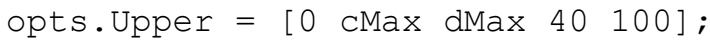

$[p, g \circ f=$ fit $(x D a t a, y D a t a$, ft, opts ) ;

catch

$\mathrm{p} \cdot \mathrm{b}=0 ; \mathrm{p} \cdot \mathrm{c}=0 ; \mathrm{p} \cdot \mathrm{d}=0 ; \mathrm{p} \cdot \mathrm{e}=0 ; \mathrm{p} \cdot \mathrm{f}=0 ;$ gof.rsquare $=0$;

end

fitData $=p \cdot c+(p \cdot d-p \cdot c) \cdot /(1+\exp (p \cdot b \cdot *(x-p \cdot e))) \cdot{ }^{\wedge} p \cdot f$;

ofill in the structure

allsamples (i).fitData=fitData;

allsamples (i).rsqr=gof.rsquare;

oNormalize by subtracting the first value

$[\mathrm{A}]=$ allsamples $(\mathrm{i})$.fitData;

$\mathrm{B}=\mathrm{A}-\mathrm{A}(1)$;

allSamples(i). Normalized=B; \%add to the structure

oconvert to the log scale

$\mathrm{B}=\log 10(\mathrm{~B})$;

allsamples (i). LogScale=B;

oidentify ct for a given threshold by interpolation

$\mathrm{RFU}=\mathrm{a}(\mathrm{z})\left(\mathrm{p} \cdot \mathrm{c}+(\mathrm{p} \cdot \mathrm{d}-\mathrm{p} \cdot \mathrm{c}) \cdot /\left(1+\exp \left(\mathrm{p} \cdot \mathrm{b} \cdot{ }^{\star}(\mathrm{z}-\mathrm{p} \cdot \mathrm{e})\right)\right) \cdot{ }^{\wedge} \mathrm{p} \cdot \mathrm{f}\right)-\mathrm{A}(1) ; \frac{\circ}{\mathrm{e}} \mathrm{eqn}$

format, baseline normalized

Zvec $=$ linspace $(1,40,1000) ;$ odefines independent var range

RFUlog=log10 (RFU (Zvec)); \%initiates Y values Zvec range in the log10

format

RFUlog=RFUlog $(R F U l o g ~>0) ;$ oadjusts the vector length to only positive values.

startVal=1000-length (RFUlog) +1 ; 


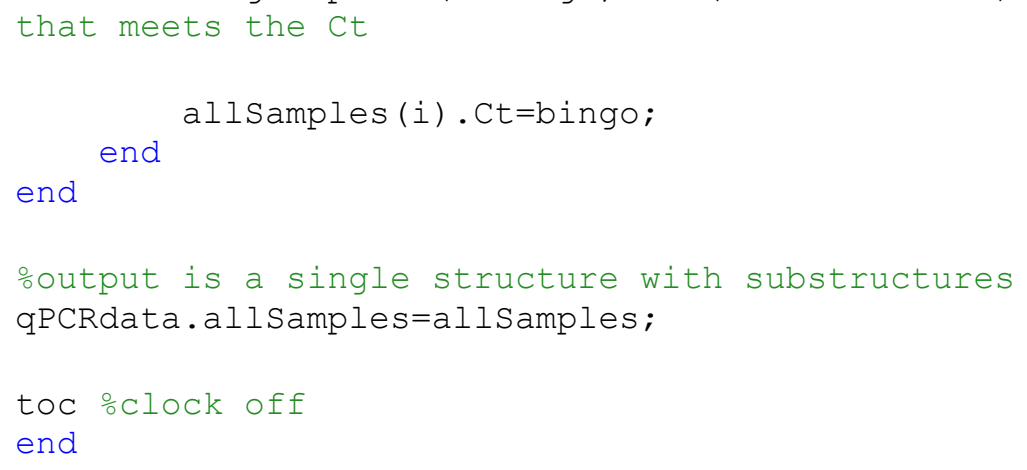


$\operatorname{REF}(7: 8,3)=\{$ qPCRdata.allSamples (31). Ct; qPCRdata.allSamples (32).Ct $\}$;

$\operatorname{REF}(9: 10,1)=\{$ qPCRdata.allSamples (41). Normalized; qPCRdata.allSamples (42) . Norma lized $\} ; 0.0001 \mathrm{X}$

$\operatorname{REF}(9: 10,2)=\{$ qPCRdata. allsamples $(41)$. LogScale;

qPCRdata.allSamples (42). LogScale; ;

$\operatorname{REF}(9: 10,3)=\{$ qPCRdata.allSamples (41).Ct; qPCRdata.allSamples (42).Ct ;

reference time 2

$\operatorname{REF}(11: 12,1)=\{$ qPCRdata.allSamples (3). Normalized; qPCRdata.allSamples (4) . Normal ized $\} ; 1 \mathrm{X}$

$\operatorname{REF}(11: 12,2)=\{$ qPCRdata.allsamples (3) . LogScale;

qPCRdata.allsamples (4). LogScale; \};

$\operatorname{REF}(11: 12,3)=\{$ qPCRdata.allSamples (3).Ct; qPCRdata.allSamples (4).Ct $\}$;

$\operatorname{REF}(13: 14,1)=\{q P C R d a t a \cdot a l l$ Samples (13). Normalized; qPCRdata.allSamples (14) . Norm alized $\} ; 0.1 \mathrm{X}$

$\operatorname{REF}(13: 14,2)=\{q P C R d a t a \cdot a l l$ Samples (13). LogScale;

qPCRdata.allsamples (14). LogScale; ;

$\operatorname{REF}(13: 14,3)=\{$ qPCRdata.allSamples (13). Ct; qPCRdata.allSamples (14).Ct $\}$;

$\operatorname{REF}(15: 16,1)=\{$ qPCRdata.allSamples (23). Normalized; qPCRdata.allSamples (24) . Norm alized $\} ; 0.01 X$

$\operatorname{REF}(15: 16,2)=\{$ qPCRdata. allSamples (23). LogScale;

qPCRdata.allSamples (24). LogScale; $;$;

$\operatorname{REF}(15: 16,3)=\{$ qPCRdata.allSamples (23). Ct; qPCRdata.allSamples (24).Ct ;

$\operatorname{REF}(17: 18,1)=\{$ qPCRdata.allSamples (33). Normalized; qPCRdata.allSamples (34). Norm alized $\}$; $0.001 \mathrm{X}$

$\operatorname{REF}(17: 18,2)=\{$ qPCRdata.allSamples (33). LogScale;

qPCRdata.allSamples (34). LogScale; ;

$\operatorname{REF}(17: 18,3)=\{$ qPCRdata.allSamples (33). Ct; qPCRdata.allSamples (34).Ct $\}$;

$\operatorname{REF}(19: 20,1)=\{$ qPCRdata.allSamples (43). Normalized; qPCRdata.allSamples (44) . Norm alized $\} ; 0.0001 \mathrm{X}$

$\operatorname{REF}(19: 20,2)=\{$ qPCRdata $\cdot$ allsamples (43). LogScale;

qPCRdata.allsamples (44). LogScale; ;

$\operatorname{REF}(19: 20,3)=\{$ qPCRdata.allSamples (43). Ct; qPCRdata.allSamples (44).Ct $\}$;

reference time 3

$\operatorname{REF}(21: 22,1)=\{q P C R d a t a \cdot a l l$ Samples (5). Normalized; qPCRdata.allSamples (6) . Normal ized $\}$; $\because \mathrm{X}$

$\operatorname{REF}(21: 22,2)=\{$ qPCRdata.allSamples (5). LogScale;

qPCRdata.allsamples (6). LogScale; ;

$\operatorname{REF}(21: 22,3)=\{$ qPCRdata.allSamples (5).Ct; qPCRdata.allSamples (6).Ct $\}$;

$\operatorname{REF}(23: 24,1)=\{q P C R d a t a \cdot a l l$ Samples (15). Normalized; qPCRdata.allSamples (16) . Norm alized $\}$; 0.1X

$\operatorname{REF}(23: 24,2)=\{q P C R d a t a \cdot a l l$ Samples (15). LogScale;

qPCRdata.allSamples (16). LogScale; ;

$\operatorname{REF}(23: 24,3)=\{$ qPCRdata.allSamples (15). Ct; qPCRdata.allSamples (16).Ct ;

$\operatorname{REF}(25: 26,1)=\{$ qPCRdata.allSamples (25). Normalized; qPCRdata.allSamples (26). Norm alized $; \div 0.01 \mathrm{X}$ 
$\operatorname{REF}(25: 26,2)=\{$ qPCRdata. allsamples $(25)$. LogScale;

qPCRdata.allsamples (26). LogScale; \};

$\operatorname{REF}(25: 26,3)=\{$ qPCRdata.allSamples (25). Ct; qPCRdata.allSamples (26).Ct $\}$;

$\operatorname{REF}(27: 28,1)=\{$ qPCRdata.allSamples (35). Normalized; qPCRdata.allSamples (36) . Norm alized $\} ; 0.001 \mathrm{X}$

$\operatorname{REF}(27: 28,2)=\{$ qPCRdata.allSamples (35). LogScale;

qPCRdata.allSamples (36). LogScale; ;

$\operatorname{REF}(27: 28,3)=\{$ qPCRdata.allSamples (35). Ct; qPCRdata.allSamples (36). Ct $\}$;

$\operatorname{REF}(29: 30,1)=\{$ qPCRdata.allSamples (45). Normalized; qPCRdata.allSamples (46) . Norm alized $\} ; 0.0001 \mathrm{X}$

$\operatorname{REF}(29: 30,2)=\{$ qPCRdata.allSamples (45). LogScale;

qPCRdata.allSamples ( 46 ). LogScale; ;

$\operatorname{REF}(29: 30,3)=\{$ qPCRdata.allSamples (45). Ct; qPCRdata.allSamples (46).Ct $\}$;

oreference time 4

$\operatorname{REF}(31: 32,1)=\{$ qPCRdata.allSamples (7). Normalized; qPCRdata.allSamples (8) . Normal ized $\}$; $\frac{\circ}{1 X}$

$\operatorname{REF}(31: 32,2)=\{$ qPCRdata.allSamples (7). LogScale;

qPCRdata.allsamples (8). LogScale; ;

$\operatorname{REF}(31: 32,3)=\{$ qPCRdata.allSamples (7).Ct; qPCRdata.allSamples (8).Ct $\}$;

$\operatorname{REF}(33: 34,1)=\{$ qPCRdata.allSamples (17). Normalized; qPCRdata.allSamples (18) . Norm alized $\} ; 0.1 \mathrm{X}$

$\operatorname{REF}(33: 34,2)=\{$ qPCRdata. allsamples $(17)$. LogScale;

qPCRdata.allSamples (18). LogScale; ;

$\operatorname{REF}(33: 34,3)=\{$ qPCRdata.allSamples (17). Ct; qPCRdata.allSamples (18).Ct $\}$;

$\operatorname{REF}(35: 36,1)=\{$ qPCRdata.allSamples (27). Normalized; qPCRdata.allSamples (28) . Norm alized $\} ; 0.01 X$

$\operatorname{REF}(35: 36,2)=\{$ qPCRdata. allsamples $(27)$. LogScale;

qPCRdata.allSamples (28). LogScale; ;

$\operatorname{REF}(35: 36,3)=\{$ qPCRdata.allSamples (27). Ct; qPCRdata.allSamples (28).Ct ;

$\operatorname{REF}(37: 38,1)=\{$ qPCRdata.allSamples (37). Normalized; qPCRdata.allSamples (38) . Norm alized $\} ; 0.001 X$

$\operatorname{REF}(37: 38,2)=\{$ qPCRdata.allSamples (37). LogScale;

qPCRdata.allSamples (38). LogScale; ;

$\operatorname{REF}(37: 38,3)=\{$ qPCRdata.allSamples (37). Ct; qPCRdata.allSamples (38).Ct $\}$;

$\operatorname{REF}(39: 40,1)=\{$ qPCRdata.allSamples (47). Normalized; qPCRdata.allSamples (48) . Norm alized $\} ; 0.0001 \mathrm{X}$

$\operatorname{REF}(39: 40,2)=\{$ qPCRdata.allSamples (47). LogScale;

qPCRdata.allsamples (48). LogScale; ;

$\operatorname{REF}(39: 40,3)=\{$ qPCRdata.allSamples (47). Ct; qPCRdata.allSamples (48).Ct $\}$;

oreferrence time 5

$\operatorname{REF}(41: 42,1)=\{$ qPCRdata.allSamples (9). Normalized; qPCRdata.allSamples (10) . Norma

lized $\}$; $\frac{\circ}{1} \mathrm{X}$

$\operatorname{REF}(41: 42,2)=\{$ qPCRdata.allSamples (9). LogScale;

qPCRdata.allsamples (10). LogScale; $;$

$\operatorname{REF}(41: 42,3)=\{$ qPCRdata.allSamples (9).Ct; qPCRdata.allSamples (10).Ct $\}$; 
$\operatorname{REF}(43: 44,1)=\{$ qPCRdata.allSamples (19). Normalized; qPCRdata.allSamples (20) . Norm alized $\} ; 0.1 \mathrm{X}$

$\operatorname{REF}(43: 44,2)=\{$ qPCRdata.allSamples (19). LogScale;

qPCRdata.allSamples (20). LogScale; ;

$\operatorname{REF}(43: 44,3)=\{$ qPCRdata.allSamples (19). Ct; qPCRdata.allSamples (20).Ct ;

$\operatorname{REF}(45: 46,1)=\{$ qPCRdata.allSamples (29). Normalized; qPCRdata.allSamples (30) . Norm alized $\} ; 0.01 X$

$\operatorname{REF}(45: 46,2)=\{$ qPCRdata.allSamples (29). LogScale;

qPCRdata.allsamples (30). LogScale; $;$

$\operatorname{REF}(45: 46,3)=\{$ qPCRdata.allSamples (29). Ct; qPCRdata.allSamples (30).Ct $\}$;

$\operatorname{REF}(47: 48,1)=\{q P C R d a t a \cdot a l l$ Samples (39). Normalized; qPCRdata.allSamples (40). Norm alized $\} ; 0.001 X$

$\operatorname{REF}(47: 48,2)=\{$ qPCRdata.allSamples (39). LogScale;

qPCRdata.allsamples (40). LogScale; ;

$\operatorname{REF}(47: 48,3)=\{$ qPCRdata.allSamples (39). Ct; qPCRdata.allSamples (40). Ct $\}$;

$\operatorname{REF}(49: 50,1)=\{$ qPCRdata.allSamples (49). Normalized; qPCRdata.allSamples (50) . Norm alized $\} ; \% 0.0001 X$

$\operatorname{REF}(49: 50,2)=\{$ qPCRdata.allSamples (49). LogScale;

qPCRdata.allSamples (50). LogScale; \}

$\operatorname{REF}(49: 50,3)=\{$ qPCRdata.allSamples (49). Ct; qPCRdata.allSamples (50). Ct $\}$;

$\div \frac{0}{0}$

o.Theta time 1

$\mathrm{TH}(1: 2,1)=\{$ qPCRdataT.allSamples (51). Normalized; qPCRdataT.allSamples (52) . Norma lized $\} ; 0.1 \mathrm{X}$

TH $(1: 2,2)=\{$ qPCRdataT.allsamples (51). LogScale;

qPCRdataT.allSamples (52).LogScale; $;$

$\mathrm{TH}(1: 2,3)=\{$ qPCRdataT.allSamples (51). Ct; qPCRdataT.allSamples (52). Ct $\}$;

TH $(3: 4,1)=\{$ qPCRdataT.allSamples $(61)$. Normalized; qPCRdataT.allSamples (62) . Norma lized $\} ; 0.01 \mathrm{X}$

$\mathrm{TH}(3: 4,2)=\{$ qPCRdataT.allsamples (61). LogScale;

qPCRdataT.allSamples (62).LogScale; ;

$\mathrm{TH}(3: 4,3)=\{$ qPCRdataT.allSamples (61). Ct; qPCRdataT.allSamples (62). Ct $\}$;

TH $(5: 6,1)=\{$ qPCRdataT.allSamples (71). Normalized; qPCRdataT.allSamples (72) . Norma lized $\} ; 0.001 \mathrm{X}$

$\mathrm{TH}(5: 6,2)=\{$ qPCRdataT.allsamples $(71)$. LogScale;

qPCRdataT.allSamples (72). LogScale; ;

$\mathrm{TH}(5: 6,3)=\{$ qPCRdataT.allSamples (71). Ct; qPCRdataT.allSamples (72). Ct $\}$;

otheta time 2

$\mathrm{TH}(7: 8,1)=\{$ qPCRdataT.allSamples (53). Normalized; qPCRdataT.allSamples (54) . Norma lized $\} ; 0.1 \mathrm{X}$

TH $(7: 8,2)=\{$ qPCRdataT.allsamples (53). LogScale;

qPCRdataT.allSamples (54).LogScale; $;$

$\mathrm{TH}(7: 8,3)=\{$ qPCRdataT.allSamples (53). Ct; qPCRdataT.allSamples (54).Ct $\}$;

$\mathrm{TH}(9: 10,1)=\{$ qPCRdataT.allSamples (63). Normalized; qPCRdataT.allSamples (64) . Norm alized $\} ; 0.01 \mathrm{X}$

TH $(9: 10,2)=\{$ qPCRdataT.allsamples $(63)$. LogScale;

qPCRdataT.allSamples (64).LogScale; ; 
$\mathrm{TH}(9: 10,3)=\{$ qPCRdataT.allSamples (63). Ct; qPCRdataT.allSamples (64). Ct $\}$;

TH $(11: 12,1)=\{$ qPCRdataT.allSamples (73). Normalized; qPCRdataT.allSamples (74). Nor malized $\}$; $0.001 X$

$\mathrm{TH}(11: 12,2)=\{$ qPCRdataT. allSamples $(73)$. LogScale;

qPCRdataT.allSamples (74).LogScale; ;

TH $(11: 12,3)=\{$ qPCRdataT.allSamples (73). Ct; qPCRdataT.allSamples (74).Ct $\}$;

을 ta time 3

$\mathrm{TH}(13: 14,1)=\{$ qPCRdataT.allSamples (55). Normalized; qPCRdataT.allSamples (56). Nor malized $\}$; $0.1 \mathrm{X}$

$\mathrm{TH}(13: 14,2)=\{$ qPCRdataT. allSamples $(55)$. LogScale;

qPCRdataT.allSamples (56).LogScale; ;

$\mathrm{TH}(13: 14,3)=\{$ qPCRdataT.allSamples (55). Ct; qPCRdataT.allSamples (56). Ct $\}$;

TH $(15: 16,1)=\{$ qPCRdataT.allSamples (65). Normalized; qPCRdataT.allSamples (66). Nor malized $\} ; 0.01 X$

$\mathrm{TH}(15: 16,2)=\{$ qPCRdataT.allSamples $(65)$. LogScale;

qPCRdataT.allSamples (66).LogScale; $;$

$\mathrm{TH}(15: 16,3)=\{$ qPCRdataT.allSamples (65). Ct; qPCRdataT.allSamples (66).Ct $\}$;

TH $(17: 18,1)=\{$ qPCRdataT.allSamples (75). Normalized; qPCRdataT.allSamples (76) . Nor malized $\} ; 0.001 X$

TH $(17: 18,2)=\{$ qPCRdataT.allSamples (75). LogScale;

qPCRdataT.allSamples (76).LogScale; \};

$\mathrm{TH}(17: 18,3)=\{$ qPCRdataT.allSamples (75). Ct; qPCRdataT.allSamples (76). Ct $\}$;

을eta time 4

TH $(19: 20,1)=\{$ qPCRdataT.allSamples (57). Normalized; qPCRdataT.allSamples (58) . Nor malized $\} ; 0.1 \mathrm{X}$

TH $(19: 20,2)=\{$ qPCRdataT.allSamples (57). LogScale;

qPCRdataT.allSamples (58).LogScale; ;

$\mathrm{TH}(19: 20,3)=\{$ qPCRdataT.allSamples (57). Ct; qPCRdataT.allSamples (58).Ct $\}$;

TH $(21: 22,1)=\{$ qPCRdataT.allSamples (67). Normalized; qPCRdataT.allSamples (68) . Nor malized $\}$; $0.01 \mathrm{X}$

$\mathrm{TH}(21: 22,2)=\{$ qPCRdataT.allSamples (67). LogScale;

qPCRdataT.allSamples (68).LogScale; ;

$\mathrm{TH}(21: 22,3)=\{$ qPCRdataT.allSamples (67). Ct; qPCRdataT.allSamples (68).Ct $\}$;

$\mathrm{TH}(23: 24,1)=\{$ qPCRdataT.allSamples (77). Normalized; qPCRdataT.allSamples (78) . Nor malized $\} ; 0.001 X$

$\mathrm{TH}(23: 24,2)=\{$ qPCRdataT.allSamples $(77)$. LogScale;

qPCRdataT.allsamples (78).LogScale; ;

TH $(23: 24,3)=\{$ qPCRdataT.allSamples (77). Ct; qPCRdataT.allSamples (78).Ct ;

을 ta time 5

$\mathrm{TH}(25: 26,1)=\{$ qPCRdataT.allSamples (59). Normalized; qPCRdataT.allSamples (60) . Nor malized $\}$; $0.1 \mathrm{X}$

TH $(25: 26,2)=\{$ qPCRdataT.allsamples (59). LogScale;

qPCRdataT.allSamples (60).LogScale; ;

TH $(25: 26,3)=\{$ qPCRdataT.allSamples (59). Ct; qPCRdataT.allSamples (60).Ct ;

$\mathrm{TH}(27: 28,1)=\{$ qPCRdataT.allSamples (69). Normalized; qPCRdataT.allSamples (70) . Nor malized $\}$; $0.01 \mathrm{X}$ 


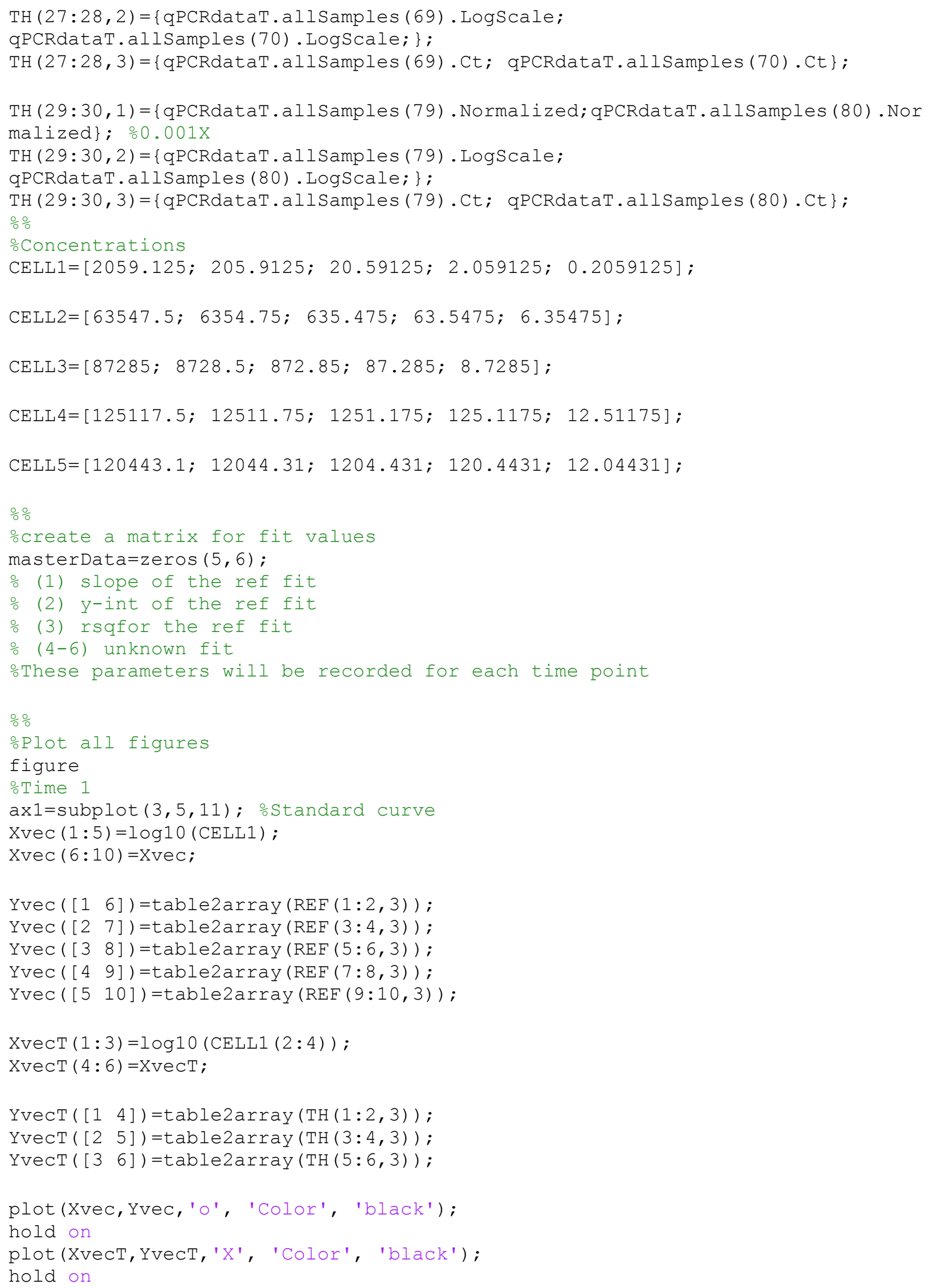




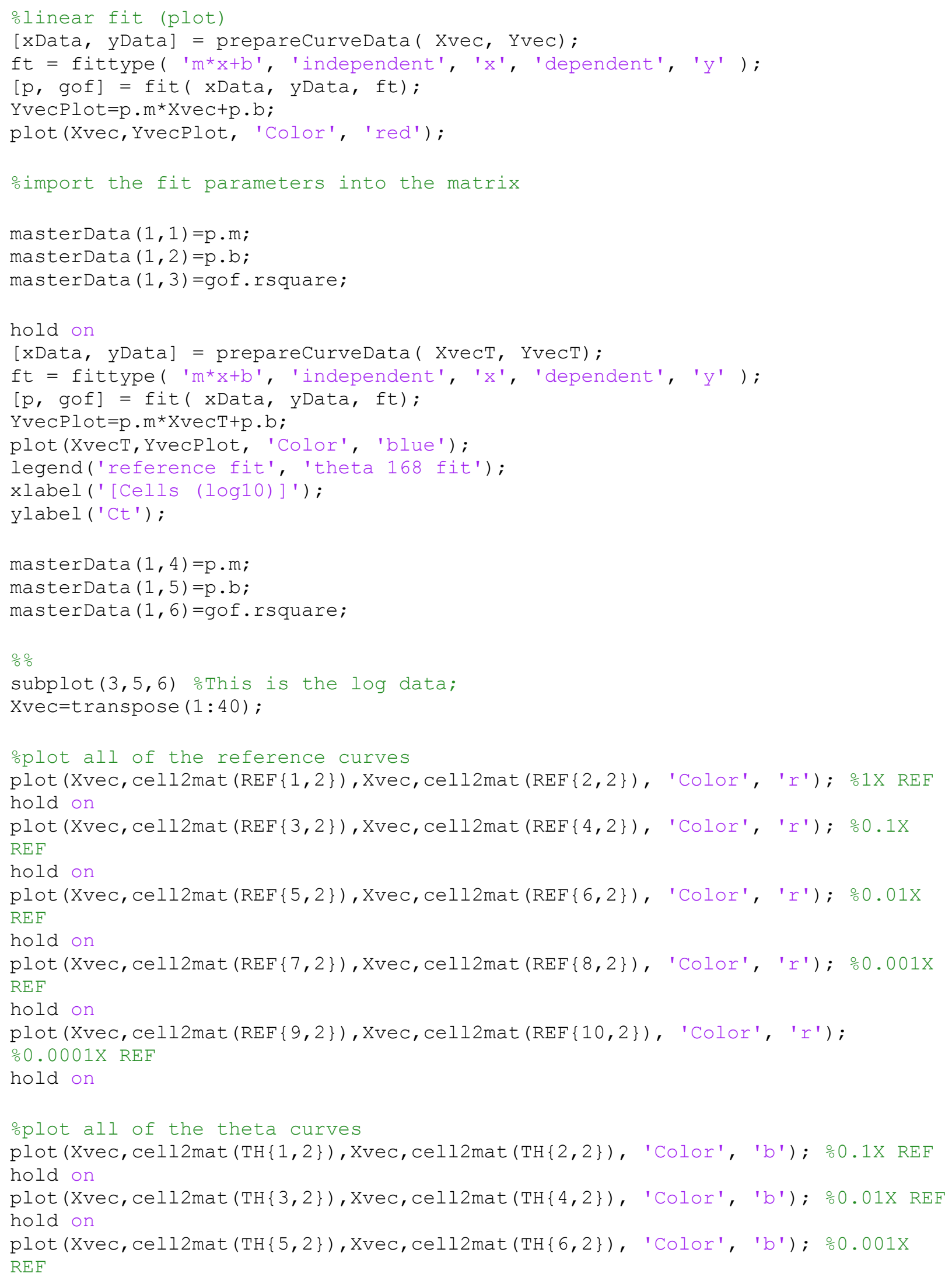




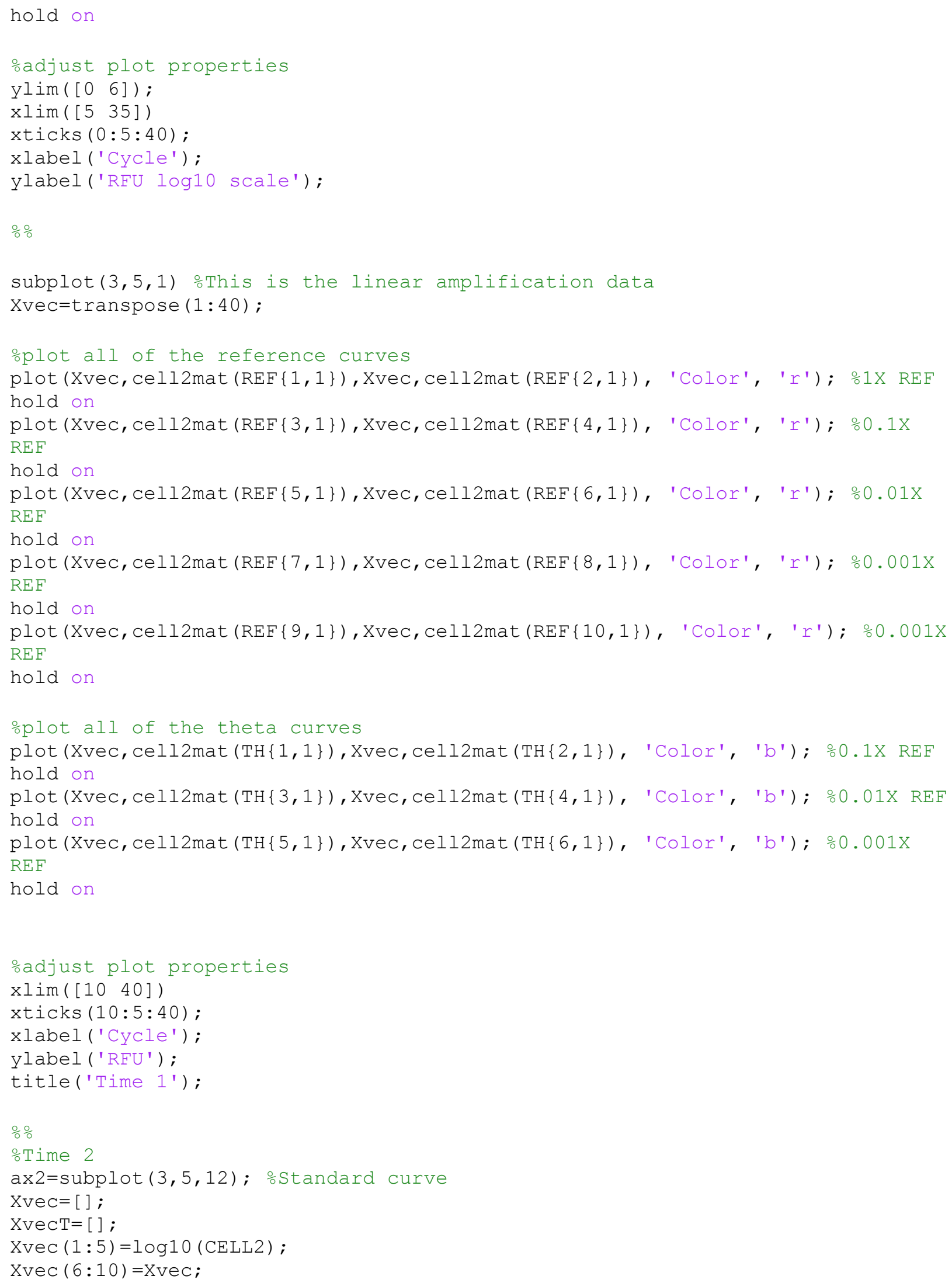




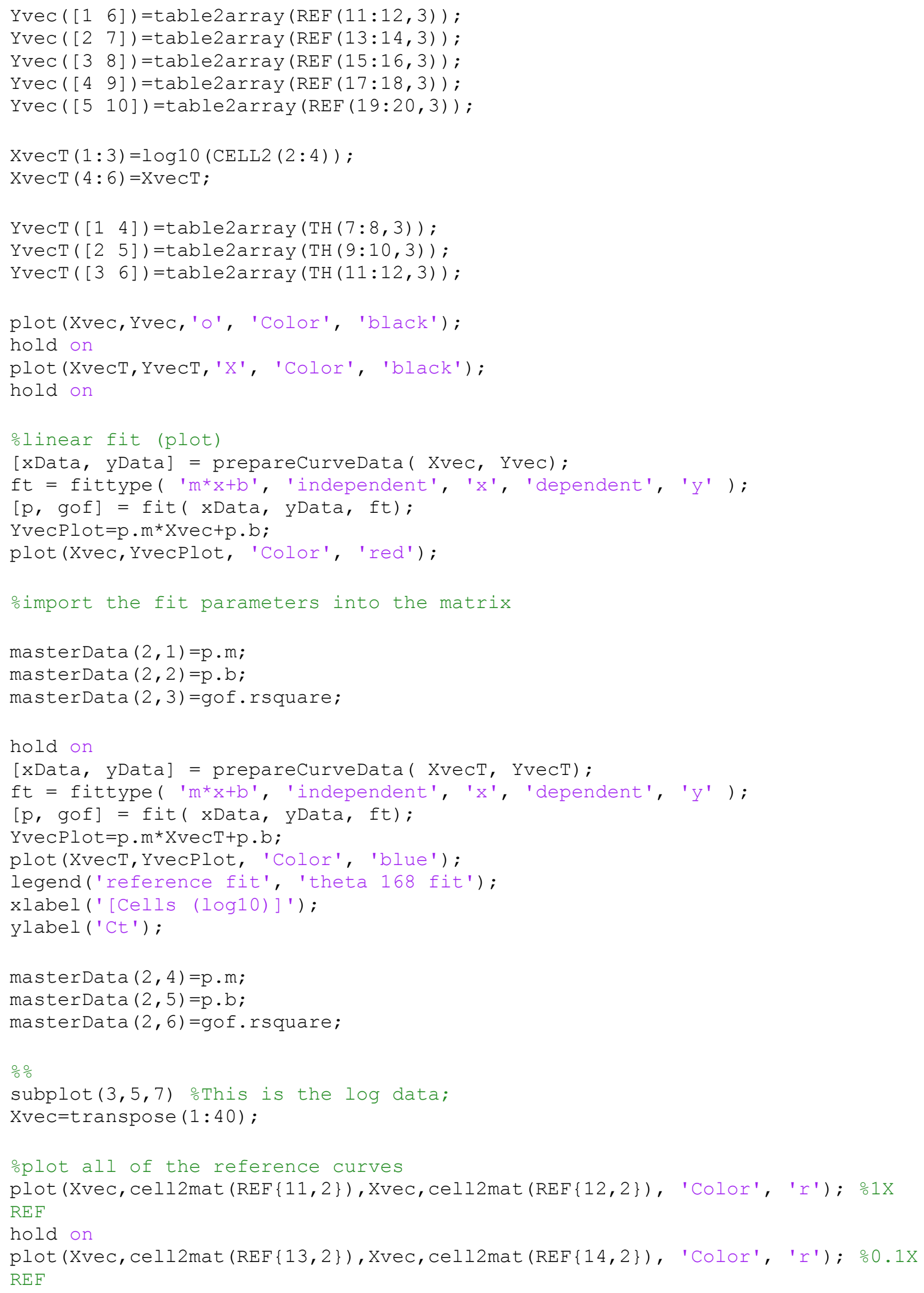




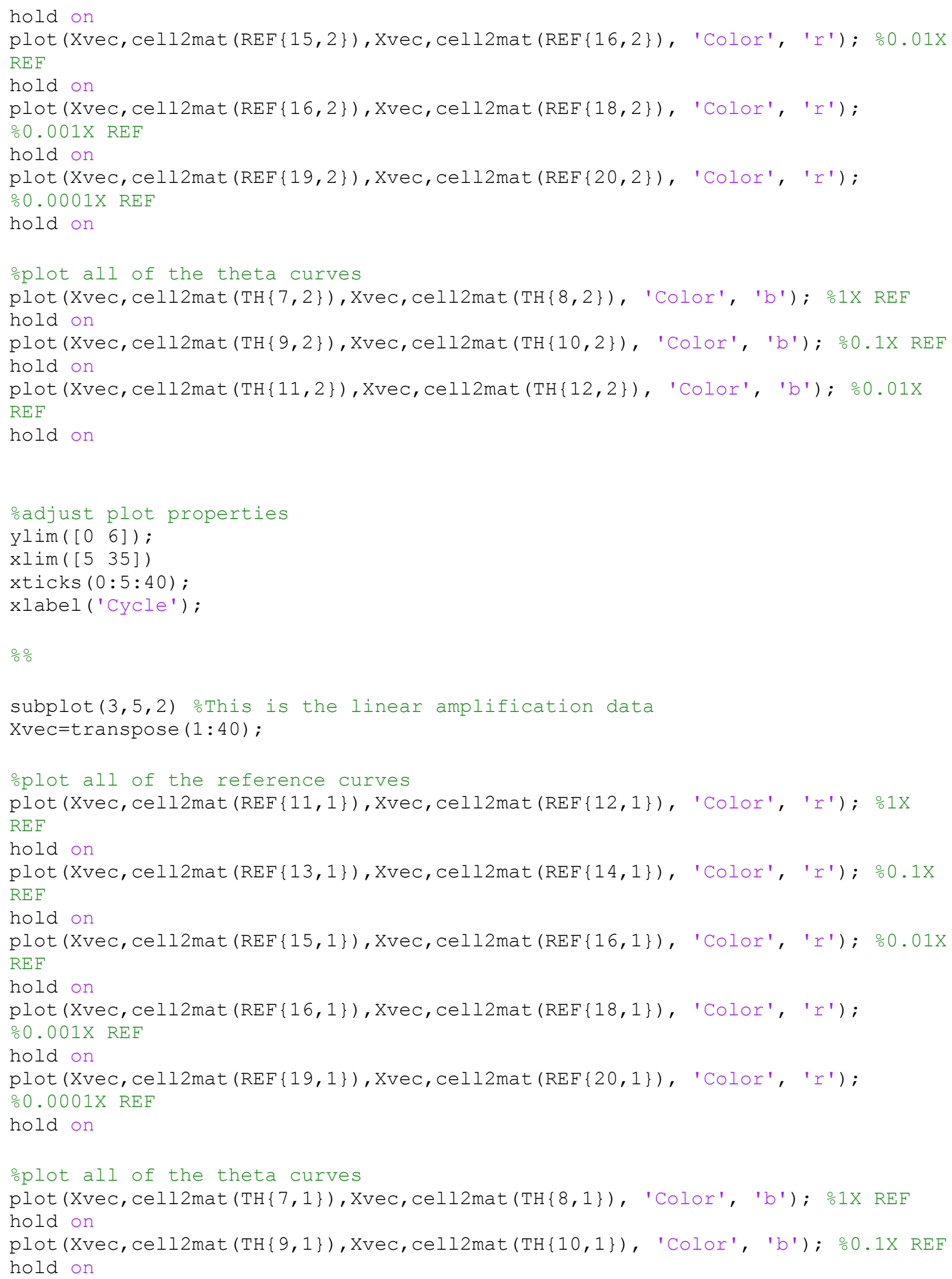




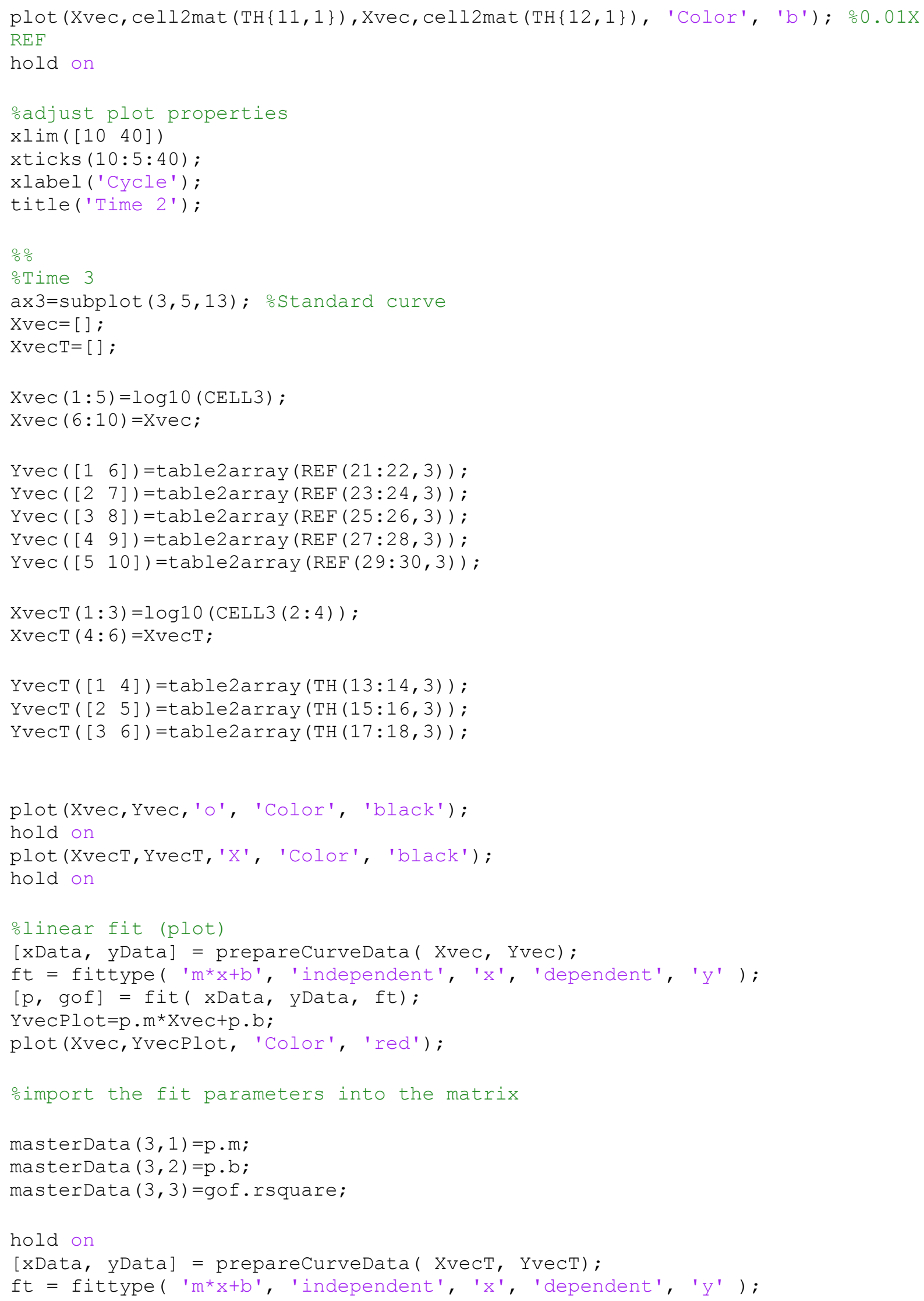




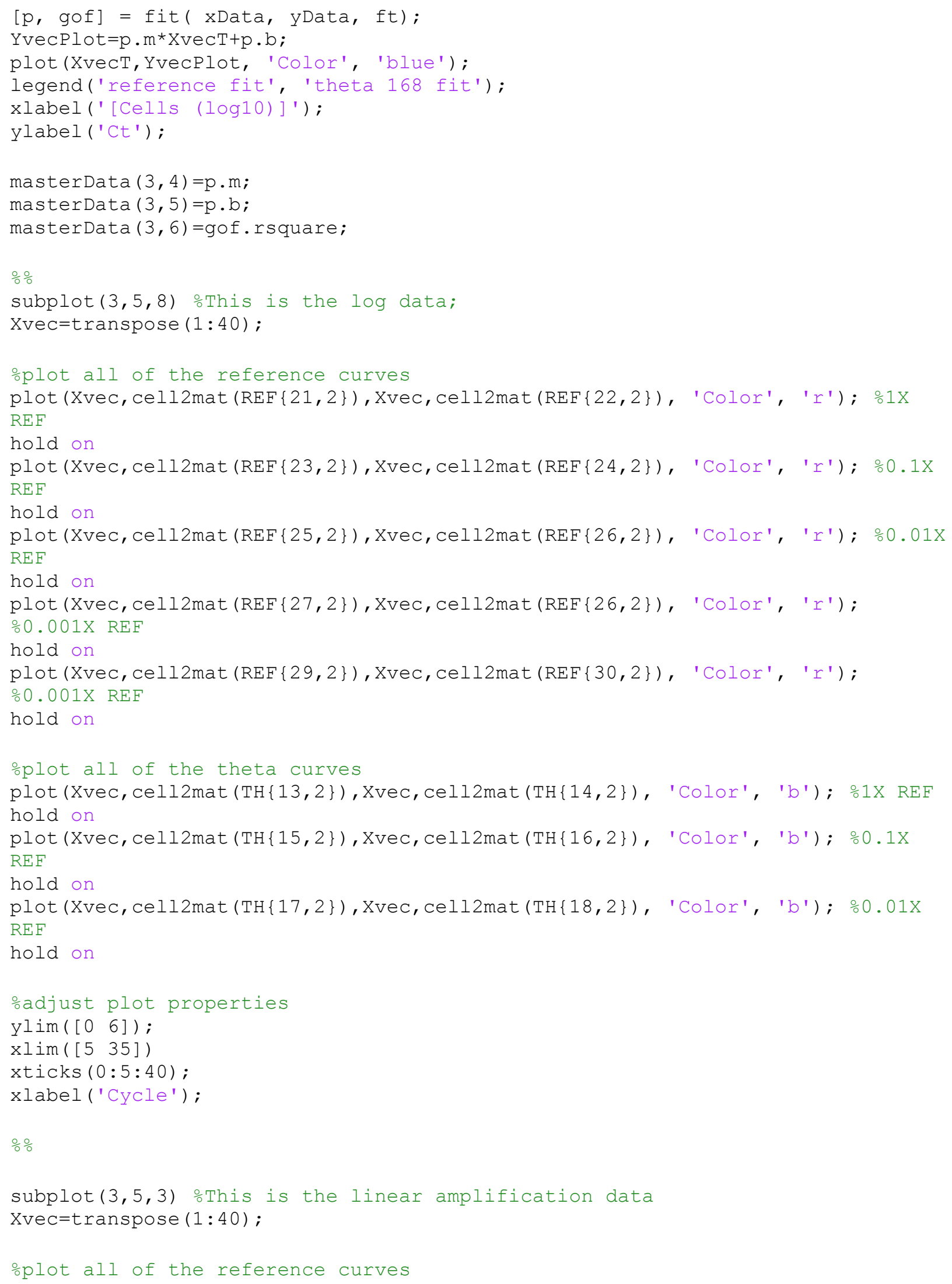




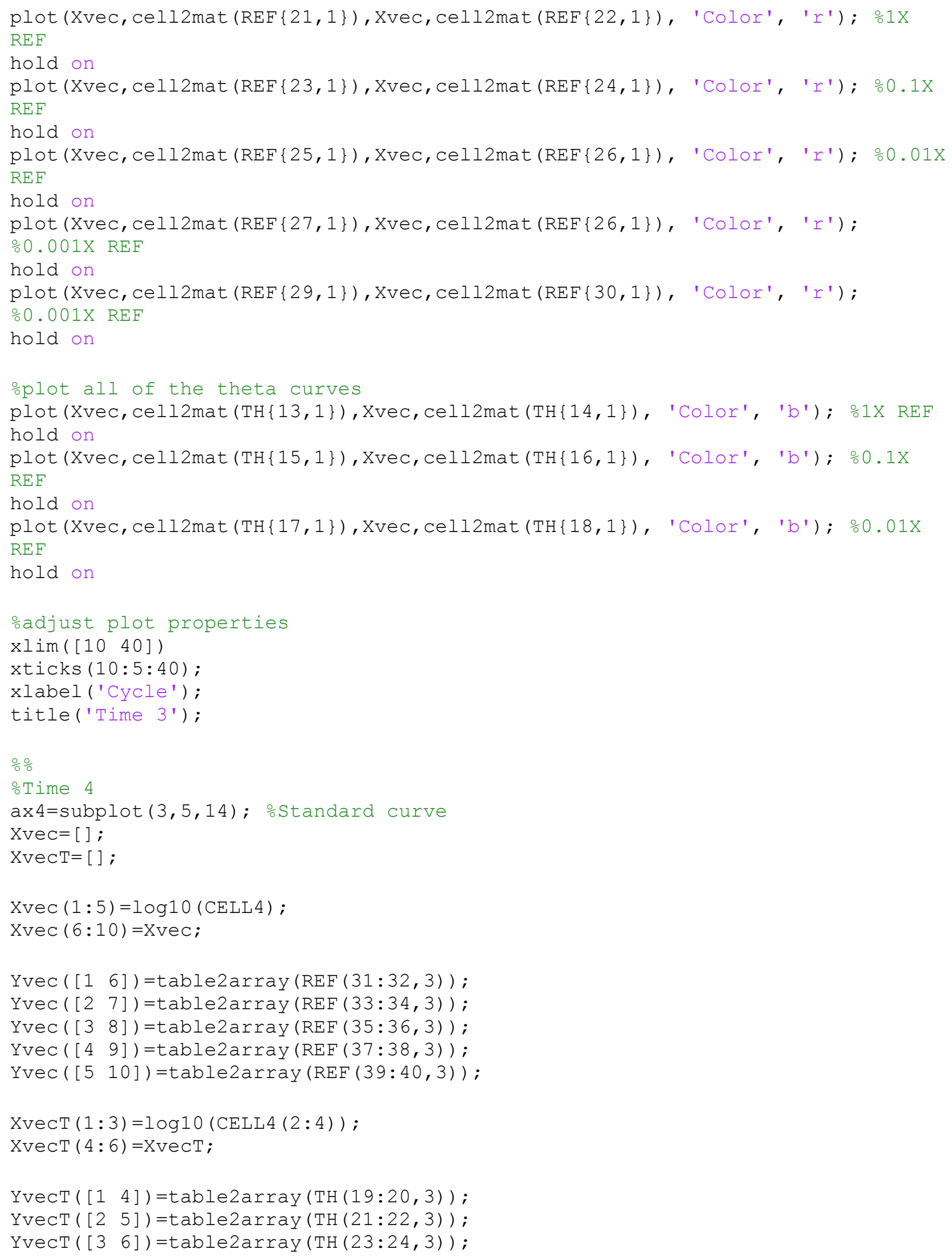




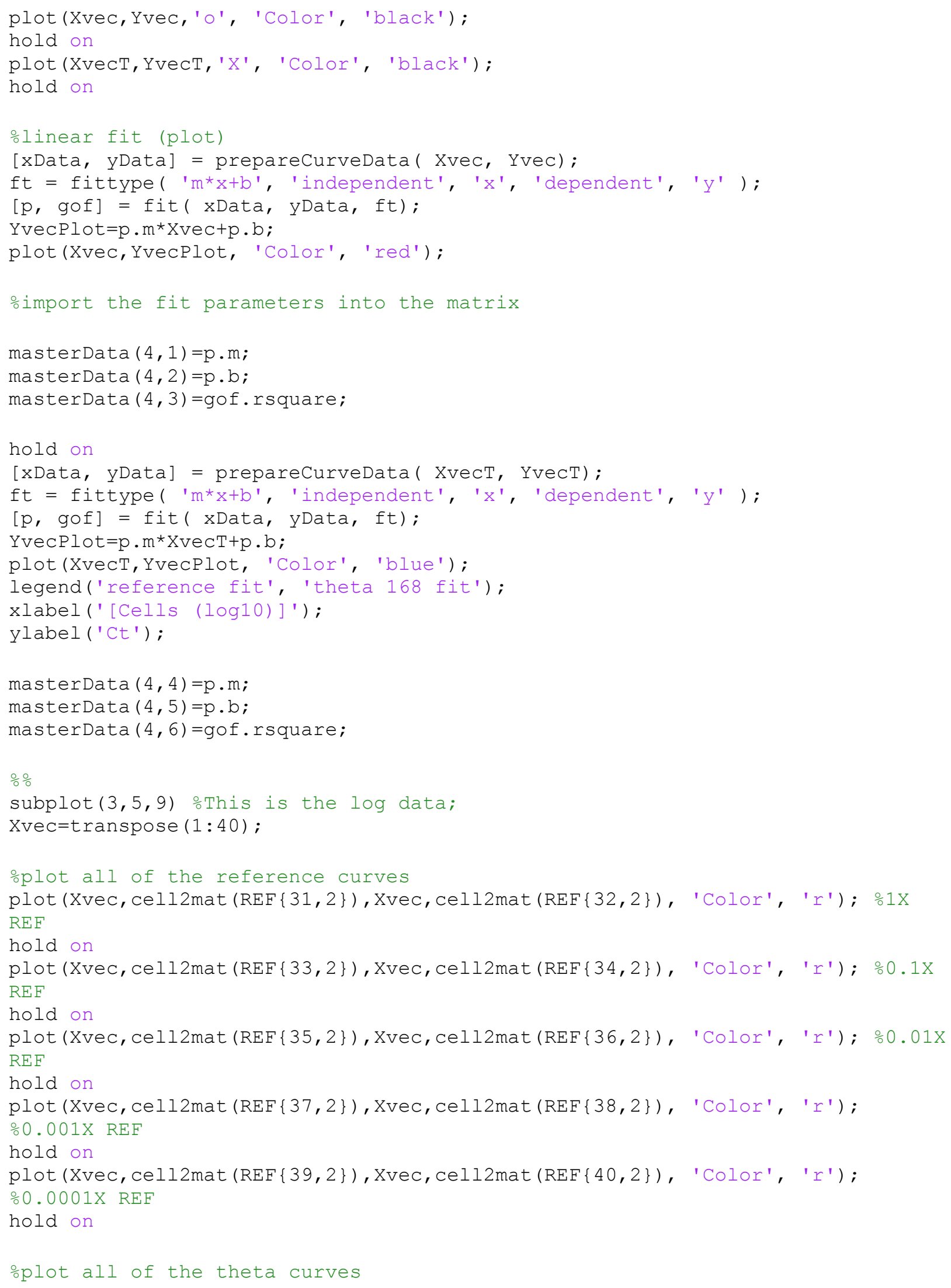




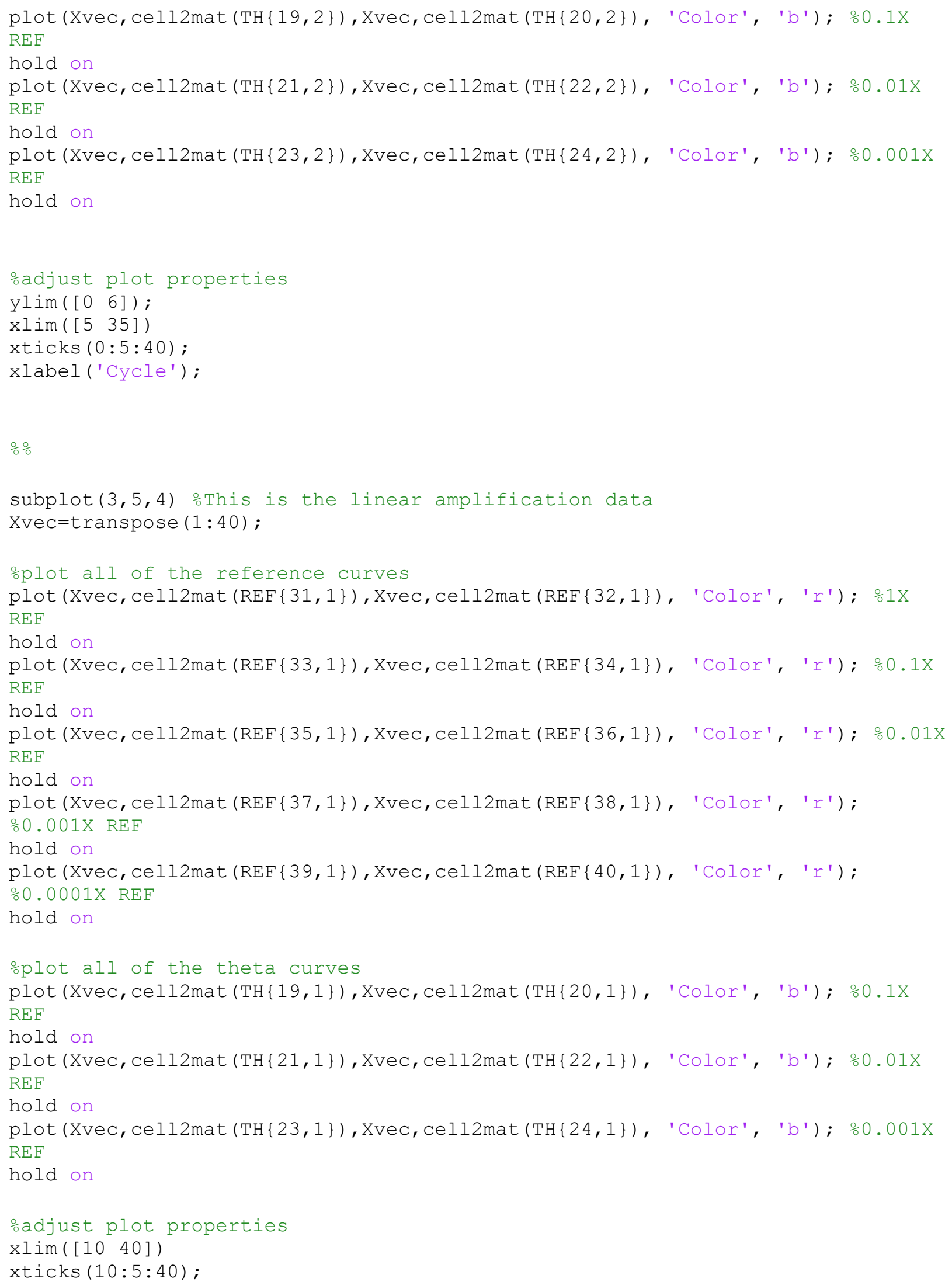




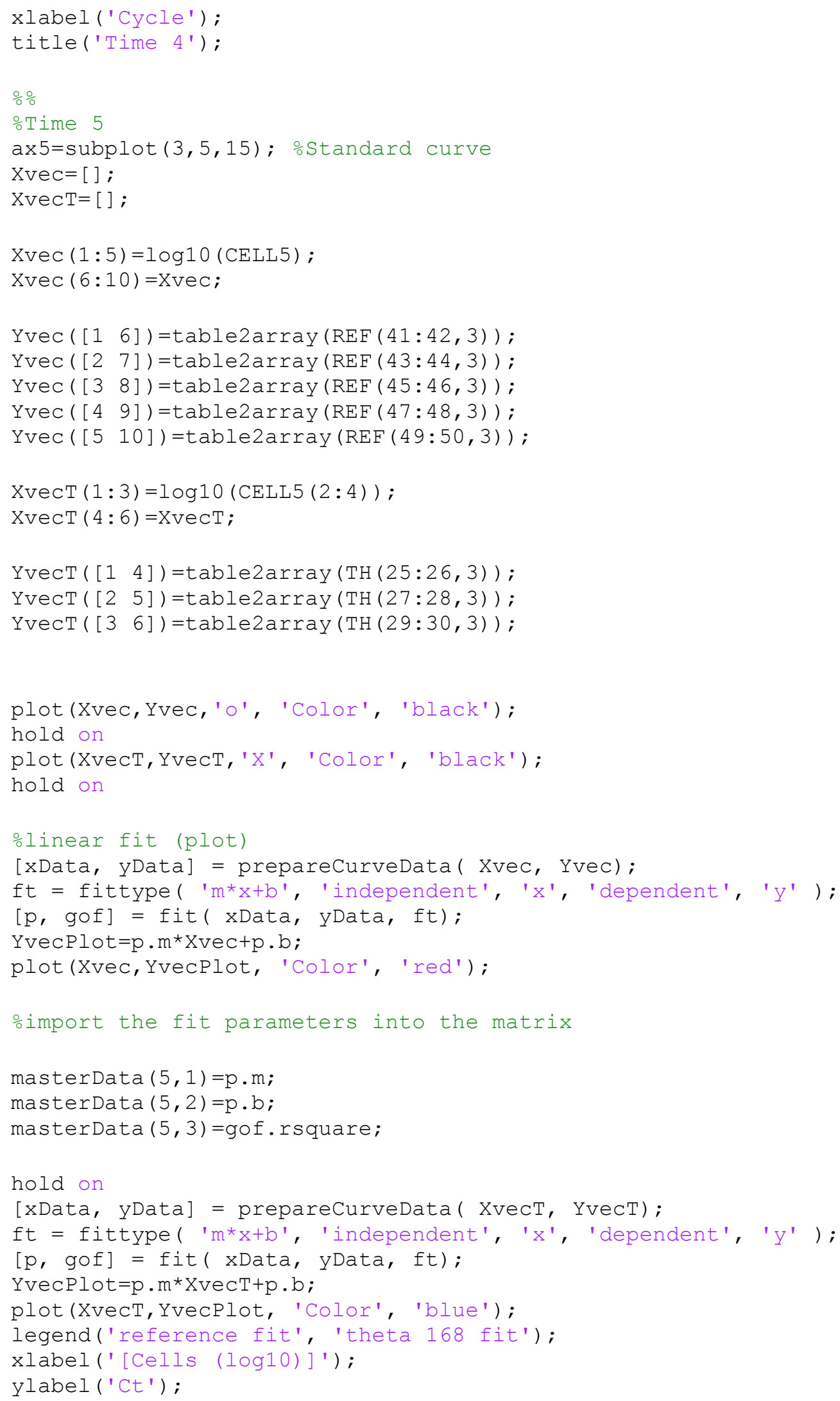




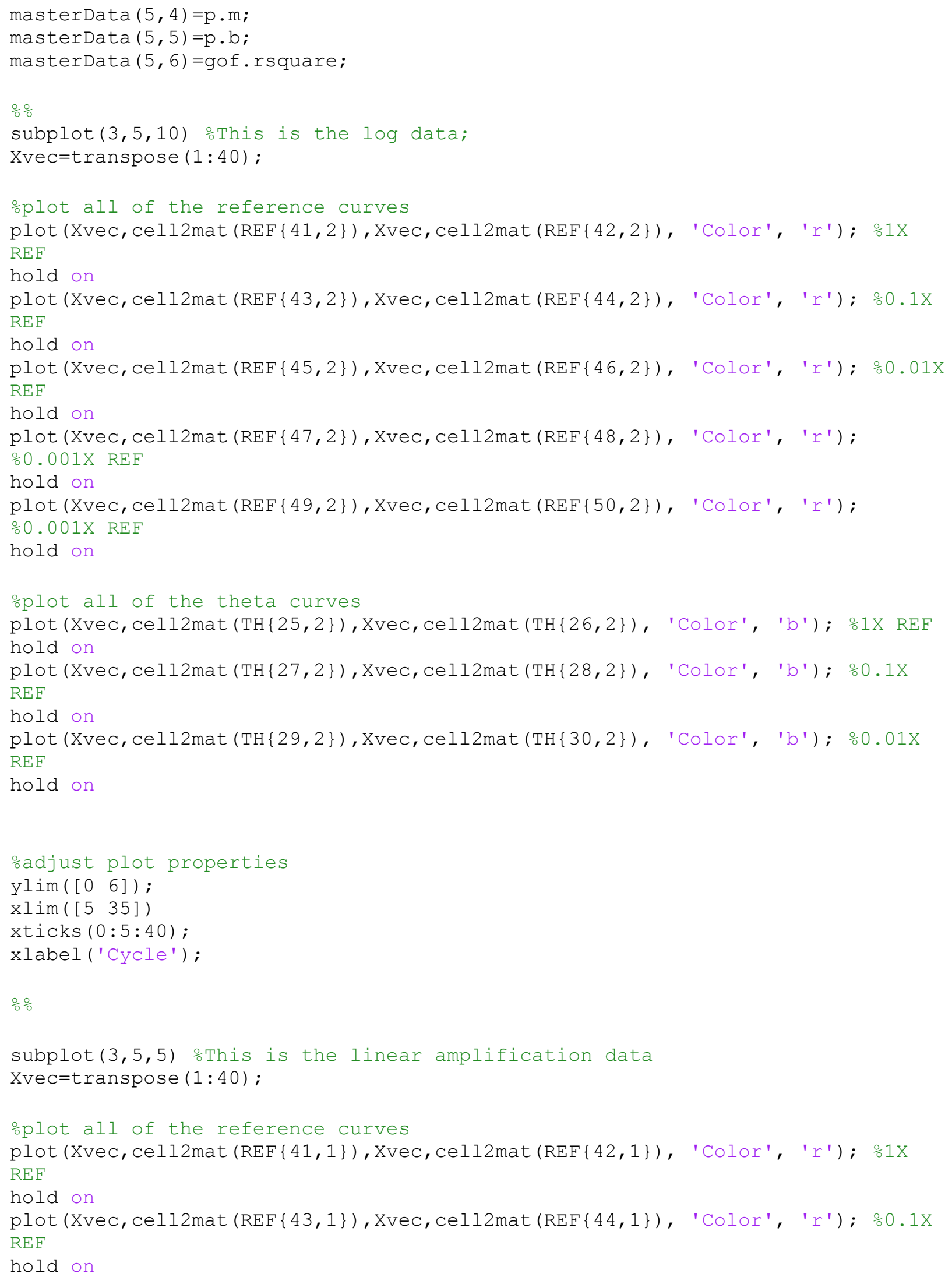




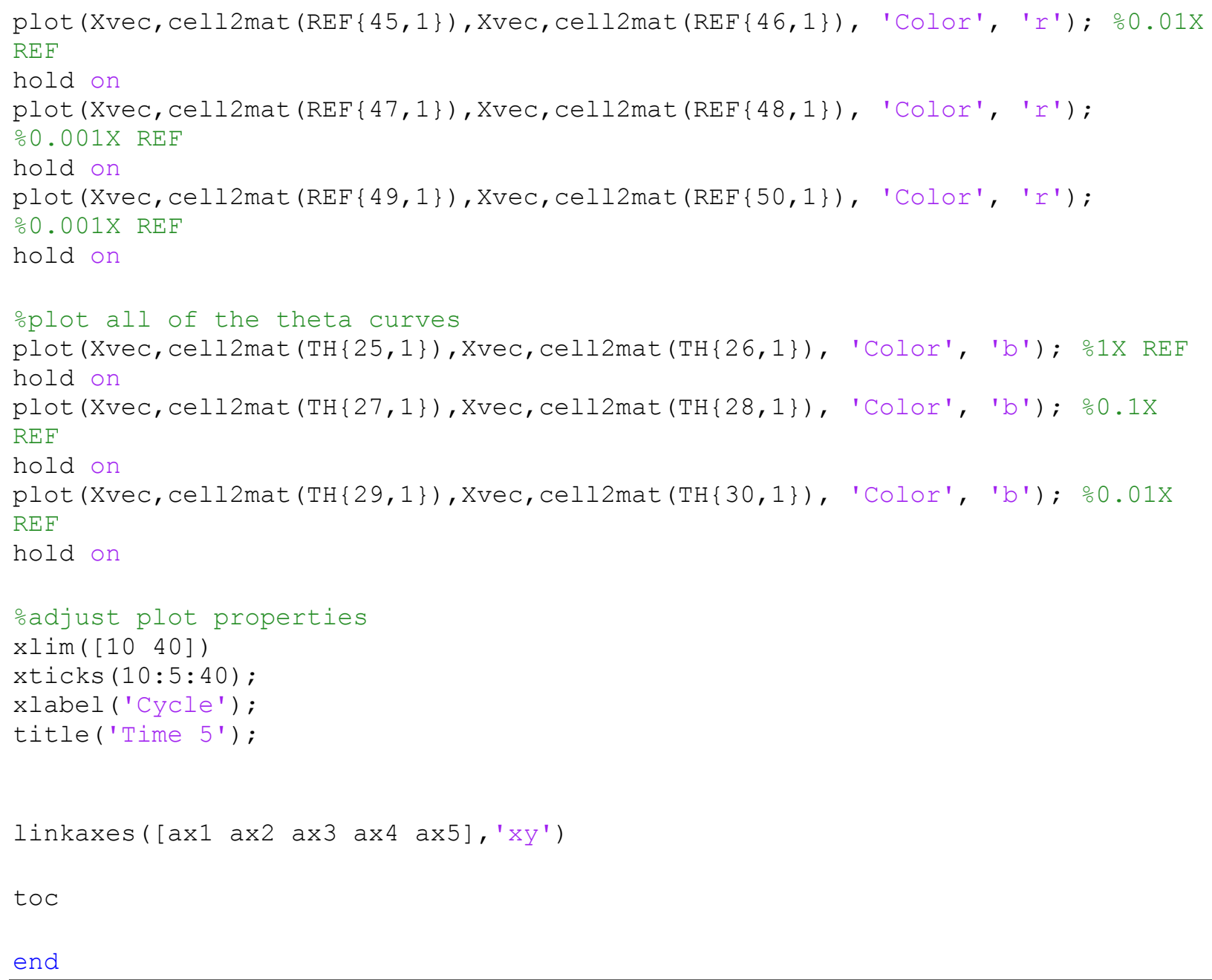

6. Bacterial Biofilm imaging.

Wild type and PolYB- B. subtilis strains with a theta replicating plasmid were grown overnight at 37C. In the morning, cells were diluted 1:100 times and applied to agarose based microscope slides. After 12 hours of incubation at $37 \mathrm{C}$ in a sealed contained with $100 \%$ humidity, microscope slides were imaged using TRITC filter set. Raw pixel intensity values were extracted from each image, and a histogram plot was created (Figure 8). Below is the code used to process these images:

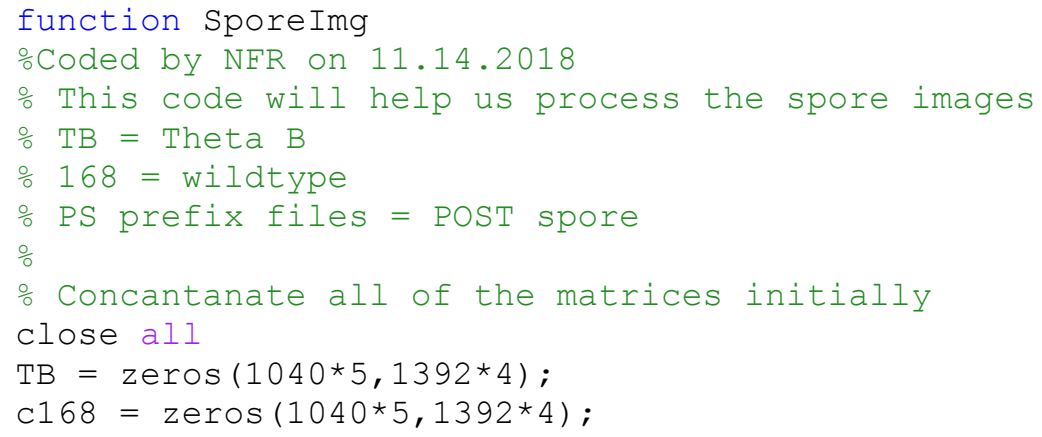




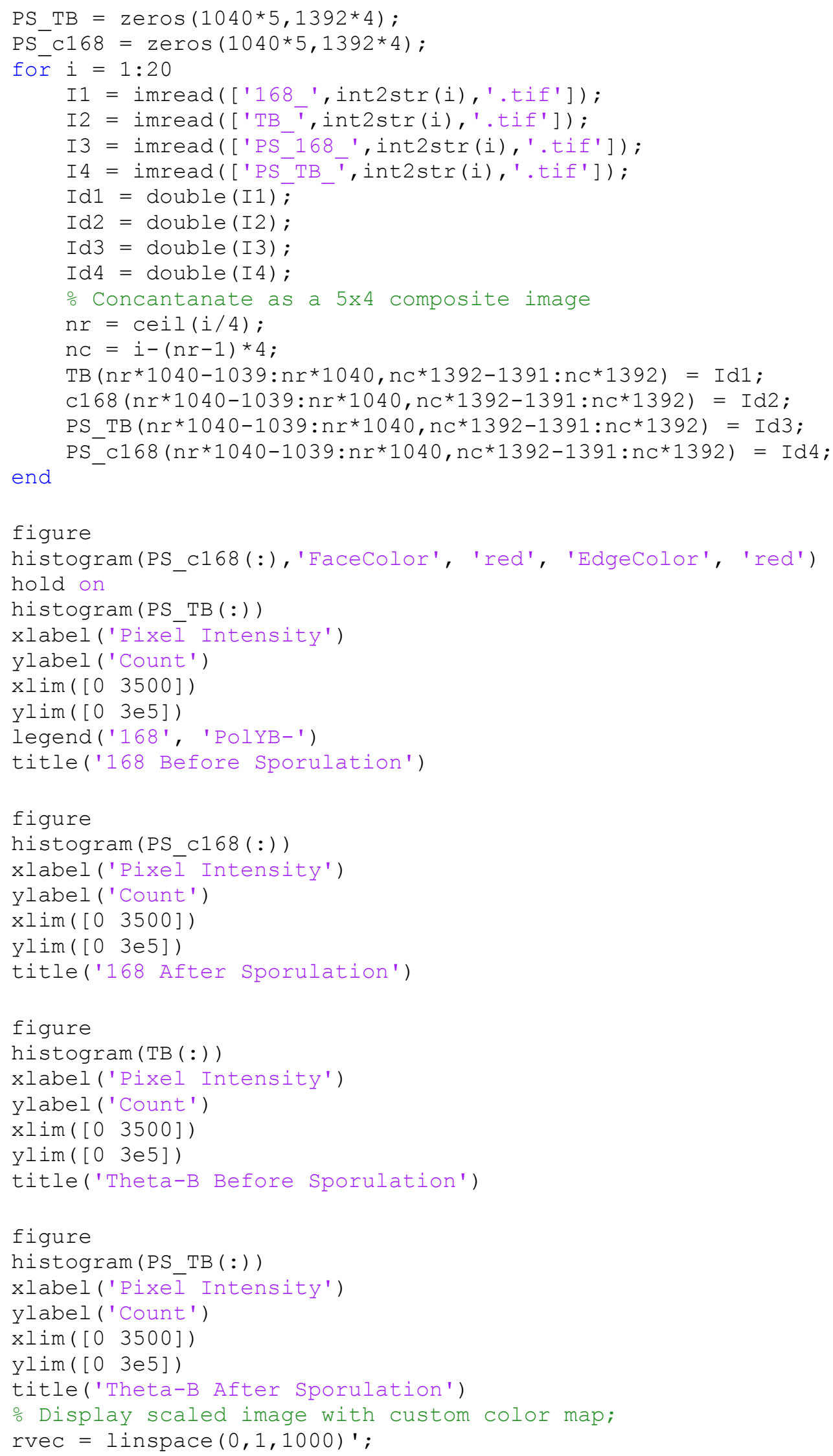




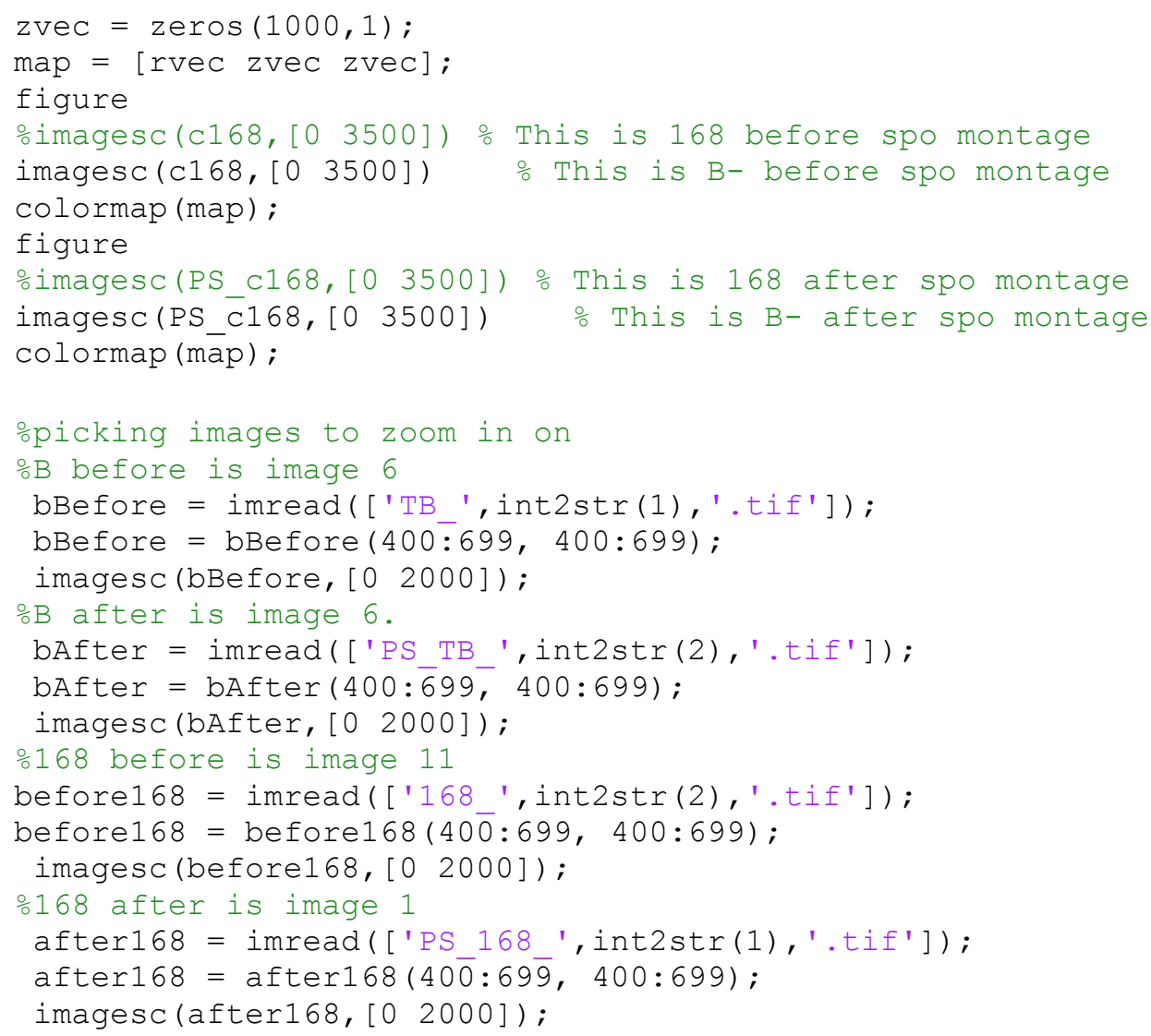

\section{Riboswitch Design and structure predictions.}

Riboswitches were designed using the riboswitch calculator (attached <Riboswitch Parameters. $x \mid s x>$ ). Six riboswitches were designed, and three of them (Theo-43, $-44,-45$ ) were selected for testing (reference the xcel file). Riboswitch Theo-45 showed the highest level of activation (Fig. 1D), and was used for all of the growth experiments. The structure of the riboswitch Theo- 45 was determined using MFold software (Supplement Figure 13). 


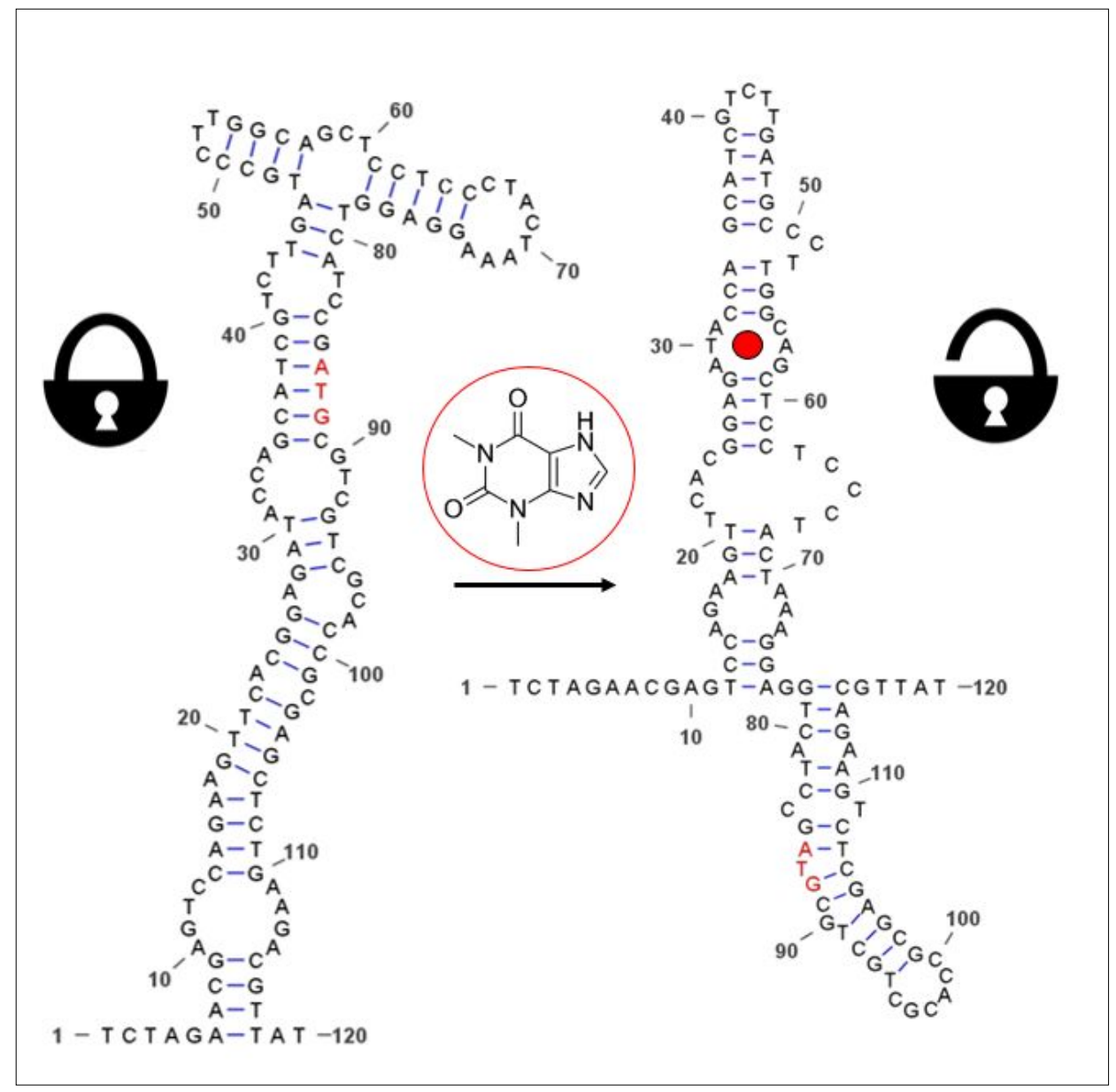

Supplement Figure 13 2D structure of the Theo-45 riboswitch generated using MFold. 